\title{
Glucose and collagen regulate human platelet activity through aldose reductase induction of thromboxane
}

\author{
Wai Ho Tang, ${ }^{1}$ Jeremiah Stitham, ${ }^{1}$ Scott Gleim, ${ }^{1}$ Concetta Di Febbo, ${ }^{2}$ Ettore Porreca, ${ }^{2,3}$ \\ Cristiano Fava, ${ }^{4}$ Stefania Tacconelli, ${ }^{3}$ Marta Capone, ${ }^{3}$ Virgilio Evangelista, ${ }^{5}$ Giacomo Levantesi, 6 \\ Li Wen,, ${ }^{7}$ Kathleen Martin,, ${ }^{1}$ Pietro Minuz,, Jeffrey Rade, ${ }^{8}$ Paola Patrignani, ${ }^{3}$ and John Hwa ${ }^{1}$ \\ ${ }^{1}$ Yale Cardiovascular Research Center, Section of Cardiovascular Medicine, Department of Internal Medicine, Yale University School of Medicine, \\ New Haven, Connecticut, USA. ${ }^{2}$ SS. Annunziata Hospital, Chieti, Italy. ${ }^{3}$ Department of Medicine, Nuroscience and Imaging, and Center of Excellence on Aging, \\ G. d'Annunzio University and "Gabriele d'Annunzio" University Foundation, CeSI, Chieti, Italy. ${ }^{4}$ Department of Medicine, University of Verona, Verona, Italy. \\ ${ }^{5}$ Laboratory of Vascular Biology and Pharmacology, Department of Translational Pharmacology Consorzio Mario Negri Sud, Santa Maria Imbaro (Chieti), Italy. \\ ${ }^{6}$ San Pio da Pietrelcina Hospital, Vasto (Chieti), Italy. ${ }^{7}$ Section of Endocrinology and Metabolism, Department of Internal Medicine, \\ Yale University School of Medicine, New Haven, Connecticut, USA. ${ }^{8}$ Division of Cardiology, Johns Hopkins School of Medicine, Baltimore, Maryland, USA
}

\begin{abstract}
Diabetes mellitus is associated with platelet hyperactivity, which leads to increased morbidity and mortality from cardiovascular disease. This is coupled with enhanced levels of thromboxane (TX), an eicosanoid that facilitates platelet aggregation. Although intensely studied, the mechanism underlying the relationship among hyperglycemia, TX generation, and platelet hyperactivity remains unclear. We sought to identify key signaling components that connect high levels of glucose to TX generation and to examine their clinical relevance. In human platelets, aldose reductase synergistically modulated platelet response to both hyperglycemia and collagen exposure through a pathway involving ROS/PLC $\gamma 2 / \mathrm{PKC} / \mathrm{p} 38 \alpha$ MAPK. In clinical patients with platelet activation (deep vein thrombosis; saphenous vein graft occlusion after coronary bypass surgery), and particularly those with diabetes, urinary levels of a major enzymatic metabolite of TX (11-dehydro-TXB 2 [TX-M]) were substantially increased. Elevated TX-M persisted in diabetic patients taking low-dose aspirin (acetylsalicylic acid, ASA), suggesting that such patients may have underlying endothelial damage, collagen exposure, and thrombovascular disease. Thus, our study has identified multiple potential signaling targets for designing combination chemotherapies that could inhibit the synergistic activation of platelets by hyperglycemia and collagen exposure.
\end{abstract}

\section{Introduction}

Accelerated atherosclerosis and microvascular disease contribute to the morbidity and mortality associated with diabetes mellitus (DM) (1-3). Vascular inflammation, endothelial dysfunction associated with hyperglycemia, impaired fibrinolysis, and increased coagulation factors as well as abnormal platelet function are typical for DM, contributing to the increased thrombotic events and development of arteriosclerosis (4). Altered platelet function in $\mathrm{DM}$, including altered adhesion and aggregation, may contribute to the pathogenesis of DM vascular complications by promoting microthrombus formation, contributing to enhanced risk of small vessel occlusions and accelerated atherothrombotic diseases $(5,6)$. Patients with type 2 DM (T2DM) exhibit platelet hyperreactivity both in vitro and in vivo, coupled with biochemical evidence of persistently increased thromboxane-dependent (TX-dependent) platelet activation $(7,8)$. Despite many important studies, the mechanism by which platelets transduce glucose levels into enhanced TX generation independently of endothelial and other blood cell-derived factors remains unclear. Similarly, optimal antiplatelet therapy for DM patients remains to be achieved.

Aldose reductase (AR) is the first enzyme of the polyol pathway, and it represents a minor source of glucose utilization, accounting for less than $3 \%$ of glucose consumption during euglycemia.

Conflict of interest: The authors have declared that no conflict of interest exists. Citation for this article: J Clin Invest. 2011;121(11):4462-4476. doi:10.1172/JCI59291.
However, during hyperglycemia, the activity of AR is substantially increased, representing up to $30 \%$ of total glucose consumption, and the abnormal activation of the polyol pathway leads to depletion of reducing equivalents and accumulation of osmotically active polyols $(9,10)$. Therefore, enhanced utilization of this pathway is well known to contribute to microvascular and macrovascular DM complications by increasing oxidative and osmotic stress. Also, the pharmacological inhibition of AR has been shown to reduce the frequency of polyneuropathy and retinopathy in DM patients (9-11). In fact, it has been shown that human AR expression in transgenic mice accelerates DM atherosclerosis (12), suggesting that AR may play an important role in atherothrombosis. We therefore hypothesized that AR contributes to platelet activation under hyperglycemic conditions and that the signaling mechanism may provide an explanation for the increased risk of atherothrombosis in DM patients.

In the present study, we investigated the role of AR in collageninduced platelet aggregation and TX biosynthesis under normalglucose (NG) and high-glucose (HG) conditions and the molecular mechanism by which AR contributes to collagen-induced platelet aggregation and TX generation. Based on these observations, we further pursued 3 case-controlled clinical studies in separate patient populations to support our mechanistic studies and to determine the clinical implications for DM patients. We demonstrate for what we believe is the first time that AR is a key transducer of hyperglycemia through a complex signaling pathway leading to 


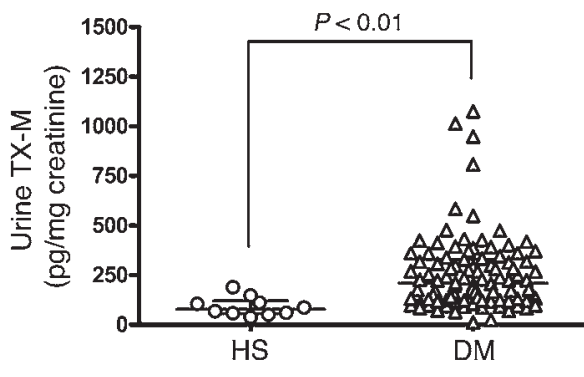

Figure 1

Levels of urinary TX-M in HS and DM patients, with all subjects on lowdose ASA (100 mg/day). The levels of urinary TX-M were measured as an index of TX release in HS $(n=10)$ and DM $(n=102)$ patients. Data are expressed as median with IQR.

the release of TX, particularly under conditions of platelet activation. Moreover, DM patients with enhanced biosynthesis of TX in vivo (as assessed by measuring a major urinary TX metabolite) despite aspirin (acetylsalicylic acid, ASA) therapy may have underlying endothelial damage and thromboembolic disease.

\section{Results}

Growing evidence has implicated hyperactive platelets and thrombus formation as critical components in the development of DM micro- and macrovascular disease. In the following studies, we systematically investigated the relationship among glucose levels, $\mathrm{TXA}_{2}$ generation, and platelet activation in human platelets and analyzed TX levels in DM patients with and without thrombosis. In addition to highlighting the importance of glucose regulation of platelet activity through $\mathrm{TXA}_{2}$ generation and release, our study identifies platelet $\mathrm{AR}$ as a key transducer of plasma glucose in regulating platelet activity.

$T X$ biosynthesis in vivo in DM patients versus non-DM patients (study group 1). The rationale for our studies was based upon our observation (Figure 1 ) and those of others $(7,8)$ of increased $\mathrm{TXA}_{2}$ generation in vivo (as measured by a major urinary TX metabolite, 11-dehydro- $\left.\mathrm{TXB}_{2}[\mathrm{TX}-\mathrm{M}]\right)$ in patients with DM. TX-M was assessed in DM patients $(n=102)$ and compared with that in a small group of normal volunteers (healthy subjects [HS]; $n=10$ ), with all subjects on low-dose ASA (selective COX-1 inhibition) reducing TXA 2 generation for prevention of cardiovascular events (13). There was a clear statistically significant increase in the DM group compared with the HS group: the median (with interquartile range [IQR; Q1-Q3]) was 77.5 (54.0 to 130.0$) \mathrm{pg} / \mathrm{mg}$ creatinine for HS and (123.4 to 330.7 ) $208.8 \mathrm{pg} / \mathrm{mg}$ creatinine for DM patients. We proceeded to study the mechanism for glucose-induced signaling on platelet $\mathrm{TXA}_{2}$ generation and activation.

Human platelet aggregation and activation are exquisitely sensitive to glucose concentrations. The effects of glucose on activation were studied in platelets isolated from venous blood of HS. The aggregation induced by $1 \mu \mathrm{g} / \mathrm{ml}$ collagen was increased when platelets were preincubated with increasing concentrations of glucose (Figure 2, A and C). Interestingly, there was no effect of glucose on ADP-induced aggregation (Figure 2, B and C), as measured by percentage of light transmission. To further validate this observation, we performed flow cytometry using P selectin antibodies and observed a similar dose-dependent effect of glucose on platelet activation in response to collagen but not ADP (Figure 2, D-F).
This highlights a sensitivity of human platelets to incremental doses of glucose $(5.5-25 \mathrm{mmol} / \mathrm{l})$ when stimulated by collagen. Important questions remained as to the mechanism and how this relates to our initial clinical observations of increased TX-M in DM patients despite the use of ASA (Figure 1). Interestingly, a recent study using $2 \mathrm{D}$ difference gel electrophoresis and mass spectrometry demonstrated that AR expression and its activity contribute to the human platelet activation after stimulation of the glycoprotein VI (GPVI) receptor by collagen (14). AR is known to metabolize glucose to sorbitol. We therefore examined the contribution of AR to collagen-induced platelet aggregation.

AR contributes to collagen-induced platelet aggregation under NG and HG conditions. We next tested the effect of 5.5 or $25 \mathrm{mmol} / 1$ glucose (basal or hyperglycemia) on human platelets in the presence or absence of epalrestat (selective AR inhibitor [ARI]). As shown in Figure 3, glucose again potentiated collagen-stimulated aggregation in human platelets. In response to $1 \mu \mathrm{g} / \mathrm{ml}$ collagen, the aggregation in $25 \mathrm{mmol} / \mathrm{l}$ glucose was $25 \%$ higher than that in $5.5 \mathrm{mmol} / \mathrm{lglucose}$ and was attenuated by treatment with $1-10 \mu \mathrm{mol} / \mathrm{l}$ epalrestat (Figure 3, A-C), suggesting that such aggregation was abolished by inhibition of AR. The concentration of epalrestat used in the present study was based on the dose-response curve (Supplemental Figure 1; supplemental material available online with this article; doi:10.1172/JCI59291DS1), which is consistent with previous studies (14). To corroborate the spectrophotometric aggregation assays, $\mathrm{P}$ selectin, the marker for platelet activation, was analyzed using flow cytometry. As in Figure 2F, collageninduced $\mathrm{P}$ selectin surface expression was enhanced in HG compared with NG (Figure 3, D-F), and epalrestat effectively attenuated the increase in $\mathrm{P}$ selectin. In addition, genetic knockdown of $A R$ reduced the $P$ selectin translocation to membrane in collagenstimulated megakaryocyte (MEG-01) platelet-like particles in NG (Supplemental Figure 2). We further reconfirmed the specificity in response to collagen activation using 2 concentrations of collagen, arachidonic acid (AA), $\mathrm{ADP}$, and thrombin receptor agonist peptide (TRAP) in addition to epalrestat (Supplemental Figure 3). These results combined suggest that AR plays a central role in collagen-induced platelet aggregation under NG, but particularly under HG, conditions. Supporting these data, we found that HG alone or collagen treatment in NG increased AR activity and that the combination of collagen and HG potentiated this effect (Figure 4A). This increase in activity could not be accounted for by increased AR expression, as collagen treatment (in NG or HG, but not $\mathrm{HG}$ alone) induced only a modest increase in AR expression (Figure 4B), which did not correlate with the degree of activation (Figure 4A). Mannitol was used as osmotic control to demonstrate that AR expression is not affected by the increased osmolarity. Thus, collagen and HG synergize in activating AR.

Inbibition of $A R$ attenuates the phosphorylation of $p 38 \alpha M A P K$ in buman platelets in response to collagen under NG and HG conditions. In order to identify potential signaling mechanisms by which AR enhances collagen-induced platelet aggregation, we performed a screen using a phosphoproteomic kinase array (R \& D Systems) (data not shown). Human platelets induced by $1 \mu \mathrm{g} / \mathrm{ml}$ collagen exhibited a 5-fold increase in p $38 \alpha$ phosphorylation, which was markedly attenuated by $10 \mu \mathrm{mol} / 1$ epalrestat. A p38 phosphoproteomic array confirmed that collagen specifically induced phosphorylation of the $\alpha$ isoforms (Figure 5A). The p38 $\alpha$ activation by collagen and inhibition by epalrestat in both NG and HG was confirmed by Western blot (Figure 5B). Interestingly, p38 $\alpha$ phosphor- 
A
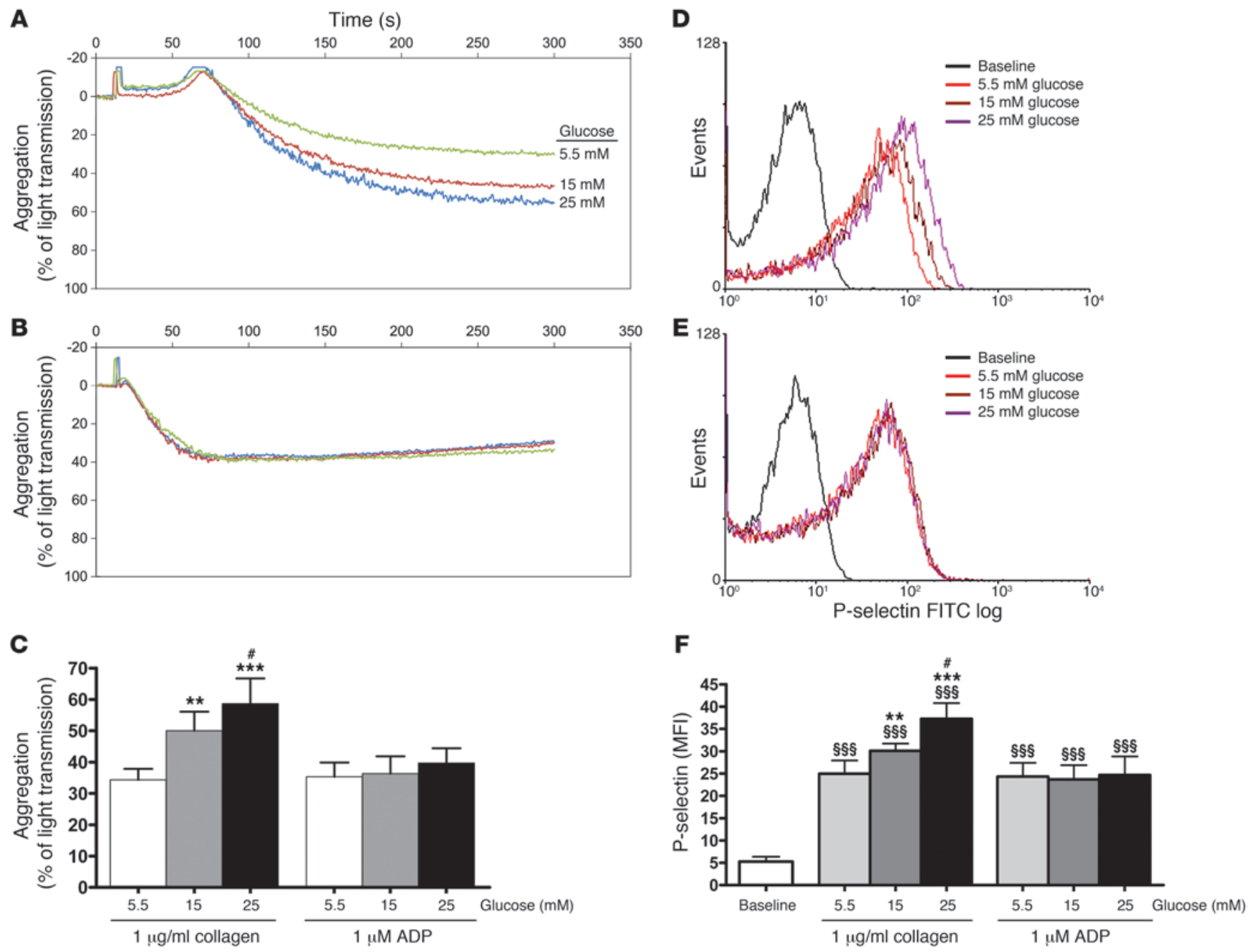

Figure 2

Glucose-induced platelet activation and aggregation in response to $1 \mu \mathrm{g} / \mathrm{ml}$ collagen or $1 \mu \mathrm{M} A D P$. The platelet suspensions were incubated with $5.5 \mathrm{mmol} / \mathrm{l}, 15 \mathrm{mmol} / \mathrm{l}$, or $25 \mathrm{mmol} / \mathrm{l}$ glucose for 90 minutes. Percentage of light transmission, an index of platelet aggregation, was measured in platelet suspensions in response to (A) $1 \mu \mathrm{g} / \mathrm{ml}$ collagen or (B) $1 \mu \mathrm{M}$ ADP for 10 minutes. (C) Quantification of data presented as percentage of light transmission. Data are expressed as mean $\pm \mathrm{SD}(n=5 \mathrm{HS})$. ${ }^{\star \star \star} P<0.001$ and ${ }^{* \star} P<0.01$ compared with $5.5 \mathrm{mM}$ glucose; ${ }^{\sharp} P<0.05$ compared with $15 \mathrm{mM}$ glucose. P selectin translocation to membrane was assessed by flow cytometry after stimulation with (D) $1 \mu \mathrm{g} / \mathrm{ml}$ collagen or (E) $1 \mu \mathrm{M}$ ADP. The representative overlay plots were presented as the number of events over the log of associated fluorescence (baseline refers to the group without collagen or ADP stimulation). (F) Quantification of data presented as MFI. Data are expressed as mean \pm SD ( $n=5 \mathrm{HS}$ ). ${ }^{* *} P<0.001$ and ${ }^{* *} P<0.01$ compared with $5.5 \mathrm{mM}$ glucose; ${ }^{\#} P<0.05$ compared with $15 \mathrm{mM}$ glucose; $\S \S P<0.001$ compared with baseline.

ylation was significantly increased in HG-incubated platelets even in the absence of collagen stimulation (Figure 5B), suggesting that $\mathrm{HG}$ incubation may potentiate platelet aggregation via $\mathrm{p} 38 \alpha$, perhaps through its downstream effector cytosolic phospholipase $\mathrm{A}_{2}$ $\left(\mathrm{CPLA}_{2}\right)(15)$, leading to TX biosynthesis. Most importantly, collagen-induced platelet aggregation in both NG and HG was reduced by pretreatment with a p38 inhibitor (Figure 5C).

We next aimed to understand the mechanism by which collagen activates p38 $\alpha$. We have demonstrated that collagen activates AR (Figure 4A). As AR can lead to ROS generation, which can regulate p38 $\alpha$ (16), we measured oxidative stress production by using specific fluorescent probes. The levels of oxidative stress and superoxide were significantly increased upon collagen-induced platelet aggregation and were even higher in HG compared with NG (Fig- ure 6A). The increases were significantly attenuated by epalrestat, suggesting that AR contributes to oxidative stress in collagenstimulated platelets. Treatment with $10 \mathrm{mmol} / \mathrm{l} \mathrm{N}$-acetyl-cysteine (NAC, ROS scavenger) or $100 \mu \mathrm{mol} / \mathrm{l}$ apocynin (NADPH oxidase inhibitor) also significantly reduced levels of oxidative stress and superoxide. Moreover, HG increased oxidative stress and superoxide production even in the absence of collagen. Notably, NAC and apocynin attenuated collagen-induced platelet aggregation and TX release to an extent similar to that of ARI, indicating that the oxidative stress likely transduced the AR signal (Figure 6, B and C). Pyocyanin (Pyo), an ROS inducer, served as a positive control in the experiment (Figure 6A).

PLC $\gamma 2$ is phosphorylated and activated in response to oxidative stress, which is thought to play an important role in plate- 
A

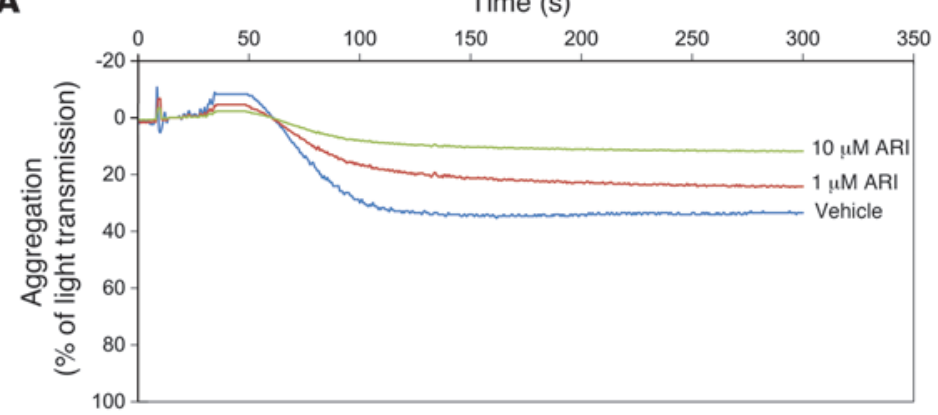

$\mathbf{B}$
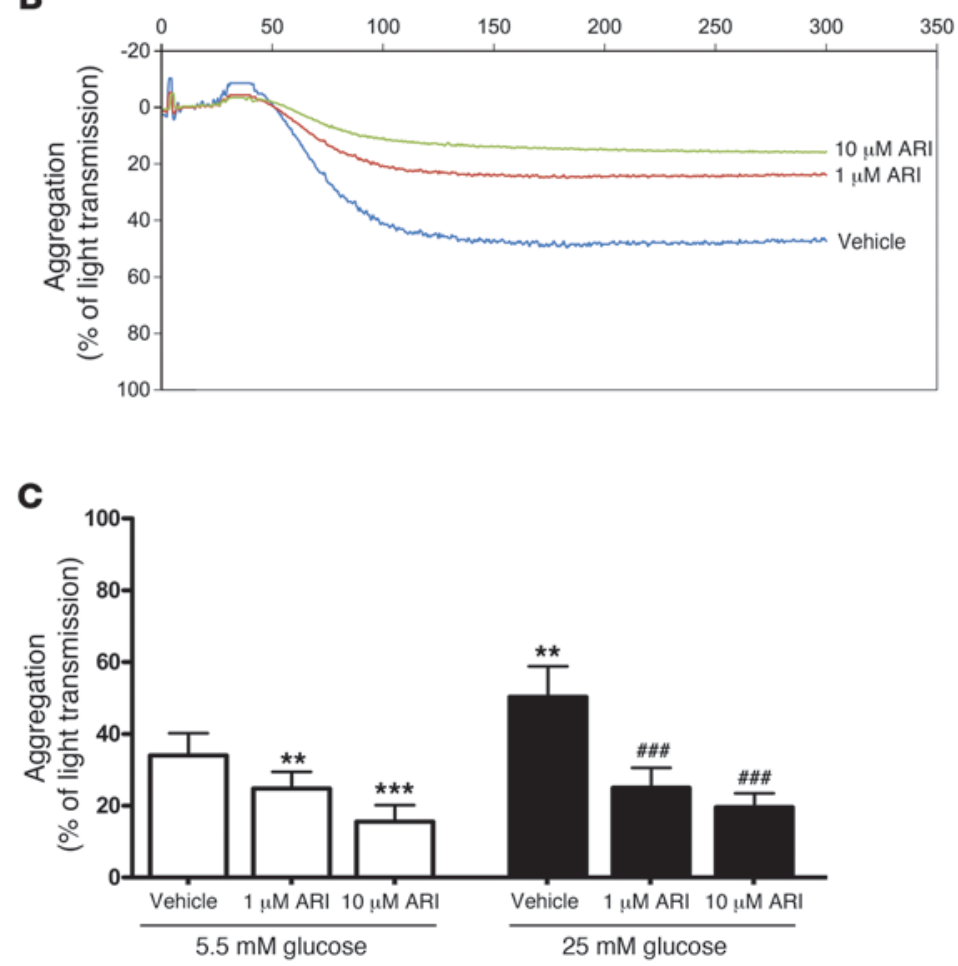

D

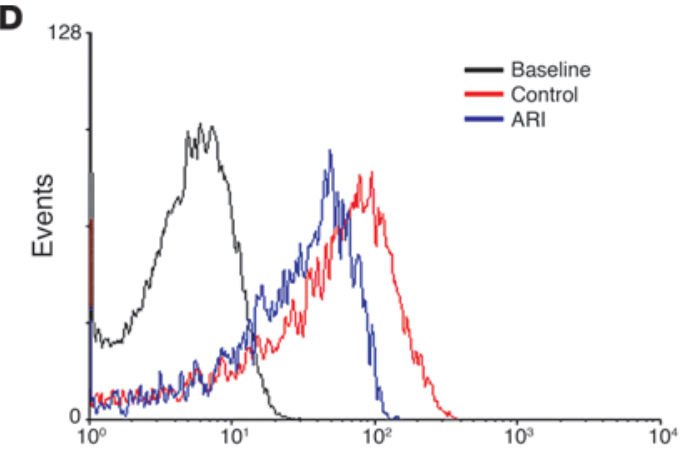

E

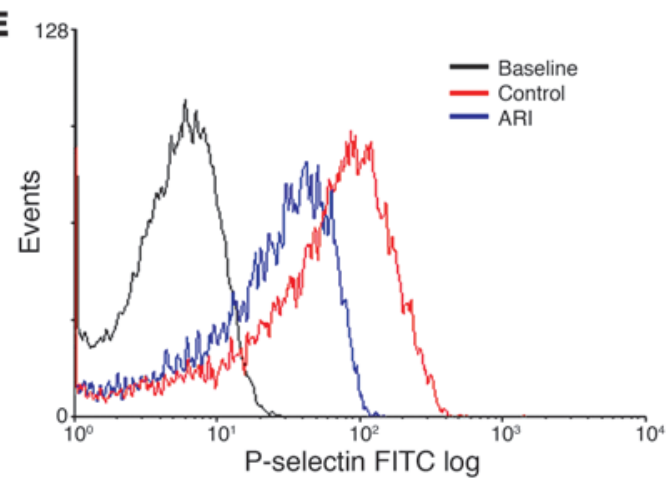

$\mathbf{F}$

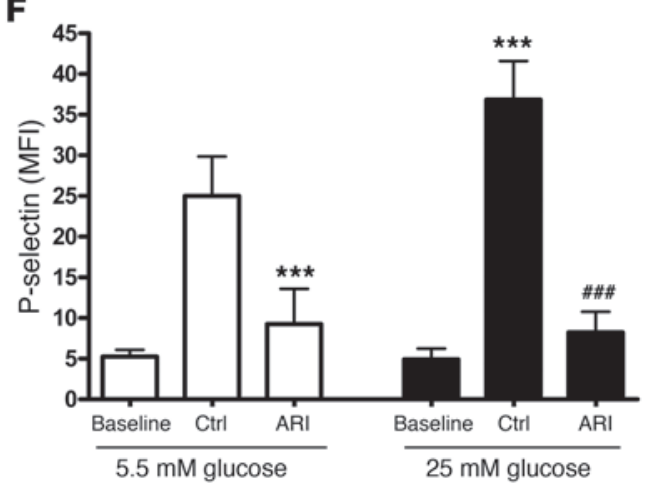

Figure 3

AR contributes to collagen-induced platelet activation and aggregation. Platelet suspensions were incubated with $5.5 \mathrm{mmol} / \mathrm{lglucose}$ (NG) or $25 \mathrm{mmol} / \mathrm{l}$ glucose $(\mathrm{HG})$ for 90 minutes in the absence (blue line) or presence of $1 \mu \mathrm{mol} / \mathrm{l}$ (red line) or $10 \mu \mathrm{mol} / \mathrm{l}$ (green line) epalrestat (ARI). Percentage of light transmission was measured in platelet suspensions under (A) NG or (B) HG conditions in response to $1 \mu \mathrm{g} / \mathrm{ml}$ collagen for 5 minutes $(n=5 \mathrm{HS}$ ). (C) Quantification of data from $1 \mu \mathrm{g} / \mathrm{ml}$ collagen expressed as aggregation in percentage of light transmission. Data are expressed as mean $\pm \mathrm{SD}(n=5 \mathrm{HS}) .{ }^{* * *} P<0.001$ and ${ }^{* *} P<0.01$ compared with the vehicle incubated in $5.5 \mathrm{mM}$ glucose; ${ }^{\# \#} P<0.001$ compared with the vehicle incubated in $25 \mathrm{mM}$ glucose. P selectin translocation to membrane was assessed by flow cytometry. Representative overlay plots in (D) NG and (E) HG groups in the presence or absence of $10 \mu \mathrm{mol} / \mathrm{l}$ epalrestat were presented as the number of events over the log of associated fluorescence (baseline refers to the group without collagen stimulation). (F) Quantification of data presented as MFI. Data are expressed as mean $\pm \mathrm{SD}(n=5 \mathrm{HS})$. ${ }^{\star \star \star} P<0.001$ compared with the control in $5.5 \mathrm{mM}$ glucose; \#\#\# $P<0.001$ compared with the control incubated in $25 \mathrm{mM}$ glucose.

let activation (17). As shown in Figure 7A, under both NG and HG conditions, the phosphorylation of PLC $\gamma 2$ was significantly enhanced upon collagen-induced platelet aggregation. Moreover, PLC $\gamma 2$ phosphorylation was increased under HG conditions even without collagen stimulation. As shown in Figure 7B, treatment with the PLC inhibitor U73122 or the ROS scavenger NAC significantly reduced collagen-induced phosphorylation of p $38 \alpha$ MAPK, providing evidence that $\mathrm{p} 38 \alpha$ MAPK activation was regulated by PLC $\gamma 2$ during collagen-induced platelet aggregation and that this process is associated with oxidative stress. In addition, treatment with another AR inhibitor, quercetin (structurally distinct from epalrestat and with broader specificity), also attenuated collageninduced phosphorylation of $\mathrm{p} 38 \alpha \mathrm{MAPK}$, further confirming the effect of AR inhibition (Figure 7B). Taken together, these results suggest that AR contributes to collagen-induced platelet activation via oxidative stress-induced tyrosine phosphorylation of PLC $\gamma 2$.

Inhibition of AR prevents the translocation and phosphorylation of $P K C$ isoforms $(\alpha, \beta I I$, and $\delta)$ in human platelets in response to collagen under $N G$ and $H G$ conditions. PLC $\gamma$ leads to the generation of diacylglycerol (DAG), which activates the PKC isoforms $(18,19)$. p38 $\alpha$ MAPK 

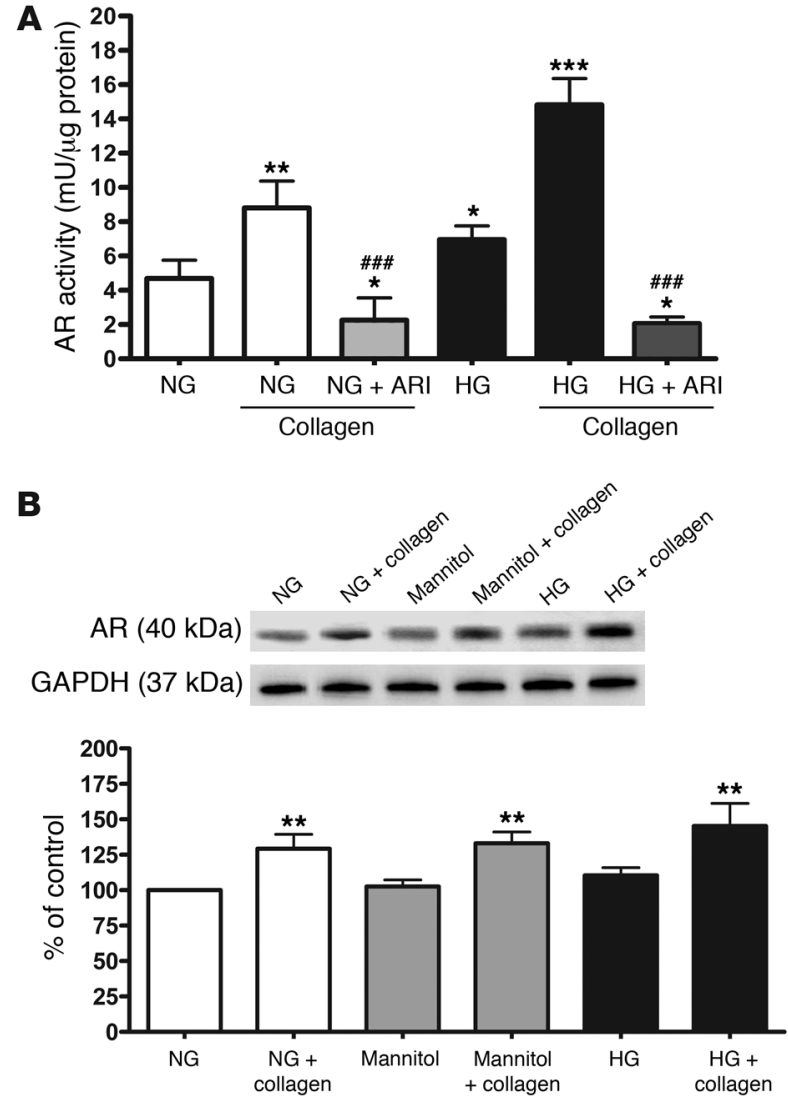

is activated downstream of PKC in platelets (20). We therefore studied the effect of AR on PKC isoforms $(\alpha, \beta I I$, and $\delta)$ known to be present in platelets. As shown in Figure 8, A and B, PKC $\alpha$ was highly expressed in the cytosolic fraction in the human platelets under both NG and HG conditions. Upon collagen stimulation, the level of PKC $\alpha$ was increased in the membrane compared with the cytosolic fraction, indicating PKC $\alpha$ translocation to a membrane during platelet aggregation. Inhibition of AR with $10 \mu \mathrm{mol} / 1$ epalrestat prevented collagen-induced translocation of $\mathrm{PKC} \alpha$ under both NG and HG conditions. Similar to PKC $\alpha, \mathrm{PKC} \beta \mathrm{II}$ and $\mathrm{PKC} \delta$ were highly expressed in the cytosolic fraction and translocated to the membrane upon collagen-induced platelet aggregation under NG conditions (Figure 8, A, C, and D). However, under HG conditions, the levels of PKC $\beta I$ II and PKC $\delta$ were higher in the membrane fraction than in the cytosolic fraction with or without $1 \mu \mathrm{g} / \mathrm{ml}$ collagen, which was attenuated by the treatment with $10 \mu \mathrm{mol} / \mathrm{l}$ epalrestat. The blots were probed with antibodies against $\mathrm{Na}^{+} / \mathrm{K}^{+}$ATPase and GAPDH, which served as the membrane and cytosolic markers, respectively (Figure 8A). Since the subcellular translocation of $\mathrm{PKC} \alpha, \mathrm{PKC} \beta \mathrm{II}$, and $\mathrm{PKC} \delta$ was prevented by AR inhibitor, we next determined whether inhibition of AR prevents the phosphorylation of PKC $\alpha$, PKC $\beta I I$, and PKC $\delta$ by using phosphospecific antibodies that correlate with kinase activity in total extracts. Consistent with the previous genetic knockout and inhibitor studies on the role of PKC isoforms in platelet aggregation $(21,22)$, the phosphorylation of different PKC isoforms was in line with the findings in their translocation (Supplemental Figure 4), indicating that $\mathrm{PKC} \alpha, \mathrm{PKC} \beta \mathrm{II}$, and $\mathrm{PKC} \delta$ translocation from cytoplasm to membrane and phosphorylation are required for colla-

\section{Figure 4}

AR activity is upregulated in collagen-stimulated platelets. (A) Activity of AR in the human platelets in response to $1 \mu \mathrm{g} / \mathrm{ml}$ collagen under $\mathrm{NG}$ and $\mathrm{HG}$ conditions. Platelet suspensions were incubated with $\mathrm{NG}$ or $\mathrm{HG}$ for 90 minutes in the presence or absence of $10 \mu \mathrm{mol} / \mathrm{l}$ epalrestat prior to stimulation by $1 \mu \mathrm{g} / \mathrm{ml}$ collagen for 10 minutes. Data are expressed as mean $\pm \mathrm{SD}(n=5 \mathrm{HS}) .{ }^{* * *} P<0.001,{ }^{* *} P<0.01$, and ${ }^{*} P<0.05$ compared with values incubated in NG alone; \#\# $P<0.001$ compared with values in $\mathrm{NG}$ and $\mathrm{HG}$ with the addition of $1 \mu \mathrm{g} / \mathrm{ml}$ collagen, respectively. (B) Expression of AR in human platelets in response to $1 \mu \mathrm{g} / \mathrm{ml}$ collagen. Platelet suspensions were incubated with NG or HG for 90 minutes prior to stimulation by $1 \mu \mathrm{g} / \mathrm{ml}$ collagen for 10 minutes, and the total cellular extract was collected for experiments. AR expression was normalized to GAPDH expression. Data are expressed as mean $\pm \mathrm{SD}(n=5 \mathrm{HS})$. ${ }^{* *} P<0.01$ compared with values incubated in NG.

gen-induced aggregation. In addition, these results suggest that HG potentiates the activation of PKC $\beta \mathrm{II}$ and $\mathrm{PKC} \delta$ similarly to the effect of collagen in an AR-dependent manner.

Inbibition of AR blocks TX release in buman platelets in response to collagen under $N G$ and $H G$ conditions. $\mathrm{CPLA}_{2}$ generates AA, the substrate for prostanoid synthesis. As $\mathrm{CPLA}_{2}$ is a downstream effector of p38 $\alpha$ MAPK in platelets $(15,16)$, we postulated that AR contributes to collagen-induced aggregation via enhanced $\mathrm{TXA}_{2}$ signaling. We assessed the generation of $\mathrm{TXB}_{2}$ (nonenzymatic metabolite of TXA $\mathrm{T}_{2}$ ) and the levels of TX receptor (TP) in the cytosolic and membrane fractions. During collagen-induced platelet aggregation, $\mathrm{TXB}_{2}$ generation was increased by 10 -fold (from $\sim 500$ to $\sim 5000 \mathrm{pg} / \mathrm{ml}$ ) under NG conditions and by nearly 12 -fold (from $\sim 650$ to $\sim 8000 \mathrm{pg} / \mathrm{ml}$ ) under $\mathrm{HG}$ conditions (Figure $9 \mathrm{~A})$. $\mathrm{TXB}_{2}$ required AR activity, as treatment with $10 \mu \mathrm{mol} / \mathrm{l}$ epalrestat significantly reduced the levels of $\mathrm{TXB}_{2}$ released from platelets. The surface expression of TP was also significantly increased, and its expression in the cytosolic fraction was decreased upon collagen-induced platelet aggregation in the HG group but not in the NG group (Figure 9B). Treatment with $10 \mu \mathrm{mol} / 1$ epalrestat significantly abolished the changes in surface expression. ROS also contributed to the $\mathrm{TXB}_{2}$ induction (Figure 6 , $A$ and $C$ ) and increased platelet activity (Figure 6B). In addition, the HG-induced surface expression of TP was also significantly attenuated by treatment with NAC and apocynin (Figure 9C), supporting a role for ROS. Collectively, these results suggest that AR and oxidative stress are required for hyperglycemia-induced TX generation and TP surface expression.

Correlation of $\mathrm{TXB}_{2}$ generation with increased platelet aggregation. We have delineated a pathway in which AR contributes to platelet activation via PLC-dependent activation of $\mathrm{p} 38 \alpha$, with subsequent $\mathrm{TXA}_{2}$ generation. To validate this pathway, platelet aggregation and $\mathrm{TXB}_{2}$ levels were measured in the presence of U73122 (PLC inhibitor), SB239063 (p38 inhibitor), quercetin, and SQ29548 (TP antagonist). Treatment with PLC and p38 inhibitors attenuated collagen-induced $\mathrm{TXB}_{2}$ release, suggesting that PLC $\gamma 2$ and its downstream effector, $\mathrm{p} 38 \alpha \mathrm{MAPK}$, contributes to $\mathrm{TXB}_{2}$ release. Moreover, all of these inhibitors reduced platelet aggregation in response to $1 \mu \mathrm{g} / \mathrm{ml}$ collagen. Importantly, treatment with SQ29548 also attenuated collagen-induced platelet aggregation, suggesting an important role for TP in increasing platelet aggregation in NG or HG (Figure 10A). SQ29548 also attenuated collagen-induced $\mathrm{TXB}_{2}$ release, suggesting a potential positive feedback mechanism (Figure 10B). Collectively, our results support a model in which AR activity increases oxidative stress and mediates PLC $\gamma 2$ 
A

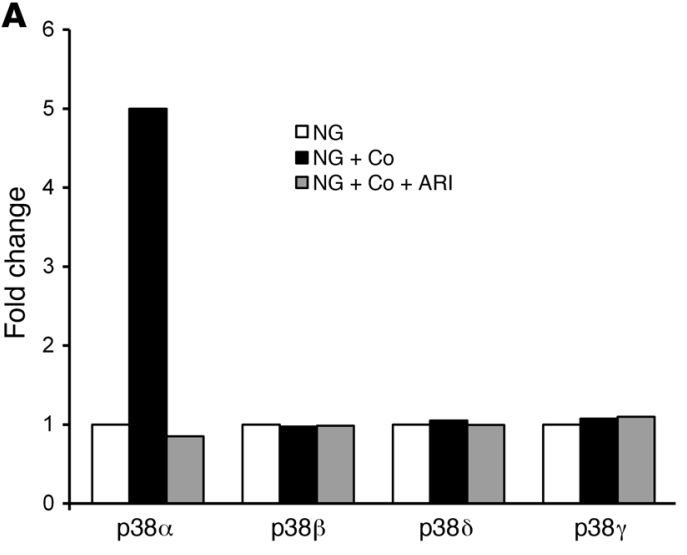

B
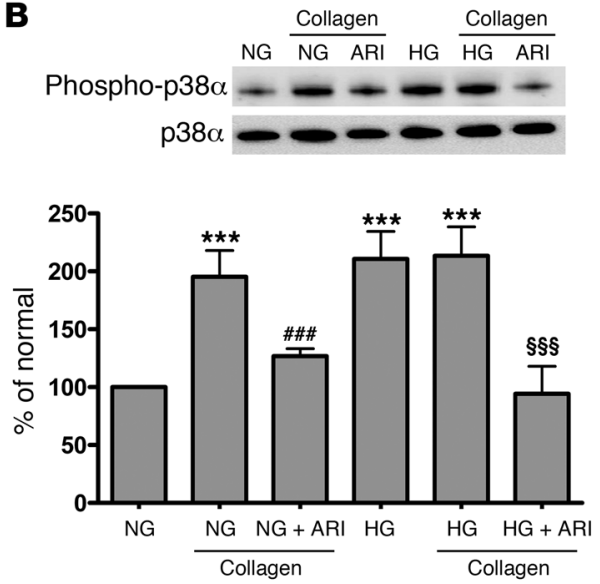

C

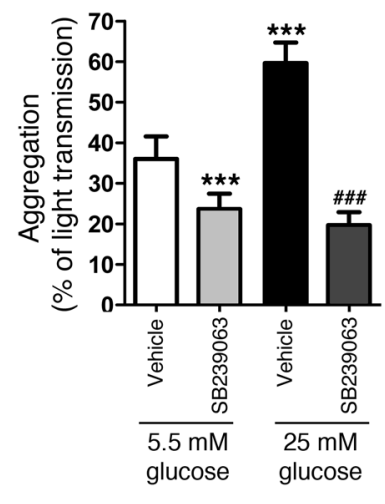

Figure 5

AR is required for p38 $\alpha$ MAPK phosphorylation in collagen-stimulated platelets. Platelet suspensions were incubated with NG or HG for 90 minutes in the presence or absence of $10 \mu \mathrm{mol} / /$ epalrestat prior to stimulation by $1 \mu \mathrm{g} / \mathrm{ml}$ collagen for 10 minutes, and the total extract was harvested for experiments. (A) Phosphorylation of different kinases in collagen-stimulated platelets under NG conditions was assayed by human phosphokinase array kits. Representative results are shown. Co, collagen. (B) Phosphorylation of p38 $\alpha$ MAPK was further measured by Western blot using specific antibody for its phosphorylated form. Data are expressed as mean $\pm \mathrm{SD}(n=5 \mathrm{HS})$. ${ }^{\star \star \star} P<0.001$ compared with values incubated in NG alone; \#\#\#<0.001 compared with values in NG with the addition of $1 \mu \mathrm{g} / \mathrm{ml}$ collagen; $\$ \S \$ P 0.001$ compared with values in $\mathrm{HG}$ with the addition of $1 \mu \mathrm{g} / \mathrm{ml}$ collagen. (C) Platelet suspensions were incubated with NG or HG for 90 minutes in the presence or absence of $50 \mu \mathrm{mol} / \mathrm{I}$ SB239063 prior to stimulation by $1 \mu \mathrm{g} / \mathrm{ml}$ collagen. Platelet aggregation was presented as the percentage of light transmission and measured in platelet suspensions under NG or HG conditions in response to $1 \mu \mathrm{g} / \mathrm{ml}$ collagen for 5 minutes. Data are expressed as mean \pm SD $(n=5 \mathrm{HS}$ ). ${ }^{\star * \star} P<0.001$ compared with vehicle incubated in NG; \#\#\# $P<0.001$ compared with vehicle incubated in HG. Gluc, glucose.

phosphorylation and PKC activation, which leads to p38 $\alpha$ MAPK activation, resulting in collagen-induced $\mathrm{TXA}_{2}$ generation, $\mathrm{TP}$ activation, and aggregation in platelets.

In order to assess the association between $\mathrm{TXA}_{2}$ generation and increased platelet aggregation, we plotted the percentage of aggregation against the level of released $\mathrm{TXB}_{2}$. As shown in Figure 10C, a linear relationship with positive slope $\left(R^{2}=0.8439\right.$; slope $\left.=6.192\right)$ occurred between $\mathrm{TXB}_{2}$ levels and the percentage of aggregation, indicating that increased platelet aggregation is closely correlated with $\mathrm{TXA}_{2}$ generation in our experimental human models. In addition, this result supports the concept that platelet aggregation increases $\mathrm{TXA}_{2}$ biosynthesis and increased $\mathrm{TXA}_{2}$ biosynthesis increases platelet aggregation, leading to an amplifying positive feedback mechanism. This important observation provides an explanation for increased platelet activity during hyperglycemia by AR-dependent induction of $\mathrm{TXA}_{2}$ generation.

Increased urinary TX-M levels in patients with deep vein thrombosis (study group 2). We demonstrated that $\mathrm{TXA}_{2}$ generation is increased with collagen-induced activation of normal human platelets ex vivo and that acute hyperglycemia further enhanced $\mathrm{TXA}_{2}$ biosynthesis in vitro. To determine whether glucose levels and collagen-dependent thrombosis induce $\mathrm{TXA}_{2}$ production in patients, we measured urinary levels of TX-M in a case-controlled study of patients with or without deep vein thrombosis (DVT). DVT is known to be caused in part by endothelial damage and collagen exposure (23). The DVT patients $(n=34)$ and controls $(n=23)$ were characterized by comparable age, cardiovascular risk factors (cigarette smoking, DM, hypertensive status, lipid levels), $\mathrm{BMI}$, and systemic inflammatory status index (i.e., C-reactive protein [CRP] and fibrinogen). As shown in Figure 11A, there was a statistically significant increase of TX-M in the DVT patients versus controls (for non-DM patients without DVT, the median [with IQR (Q1-Q3)] was 551.5 [436.2 to 600] pg/mg creatinine; for non-DM patients with DVT, it was 942 [657.5 to 1319] pg/mg creatinine). There were $8 \mathrm{DM}$ patients out of 57 total patients, but unfortunately the data on urinary levels of TX-M were only available in 5 DM patients. Interestingly, these few DM patients that were presented had very high TX-M only in the presence of DVT. A further study was necessary in a patient population with a higher incidence of DM to assess the link among DM (hyperglycemia), thrombosis (platelet hyperactivity), and $\mathrm{TXA}_{2}$ generation in vivo (urinary levels of TX-M).

Increased urinary levels of TX-M in DM patients with saphenous vein coronary artery bypass graft thrombosis treated with ASA (study group 3). Because of our interesting DVT findings, we performed another casecontrolled study assessing another patient population with a much higher prevalence of DM: a severe coronary artery disease population with coronary artery bypass graft (CABG) thrombosis (Table 1). Not surprisingly, with such severe disease, all patients were on 325 mg daily ASA (to inhibit platelet COX-1-dependent production of $\mathrm{TXA}_{2}$ ) for secondary prevention of further cardiovascular events. The groups were comparable for age and cardiovascular risk factors. As shown in Figure 11B, there were no TX-M differences between DM and non-DM patients without graft occlusion while on ASA (for non-DM patients without CABG thrombosis, the median [with IQR (Q1-Q3)] was $328 \mathrm{pg} / \mathrm{mg}$ [233 to 424.5] creatinine; for DM patients without CABG thrombosis, it was $312 \mathrm{pg} / \mathrm{mg}$ [226 and 495] creatinine). However, among those with thrombosis, there was a significant difference between DM and non-DM patients (for non-DM patients with CABG thrombosis, the median [with IQR (Q1-Q3)] was 304 [206 and 450] pg/mg creatinine; for DM patients with CABG thrombosis, it was 466.5 [275 and 718] pg/mg creatinine). This supports our experimental observation that $\mathrm{TXA}_{2}$ biosynthesis in vivo is enhanced in patients with both thrombosis and DM despite the use 

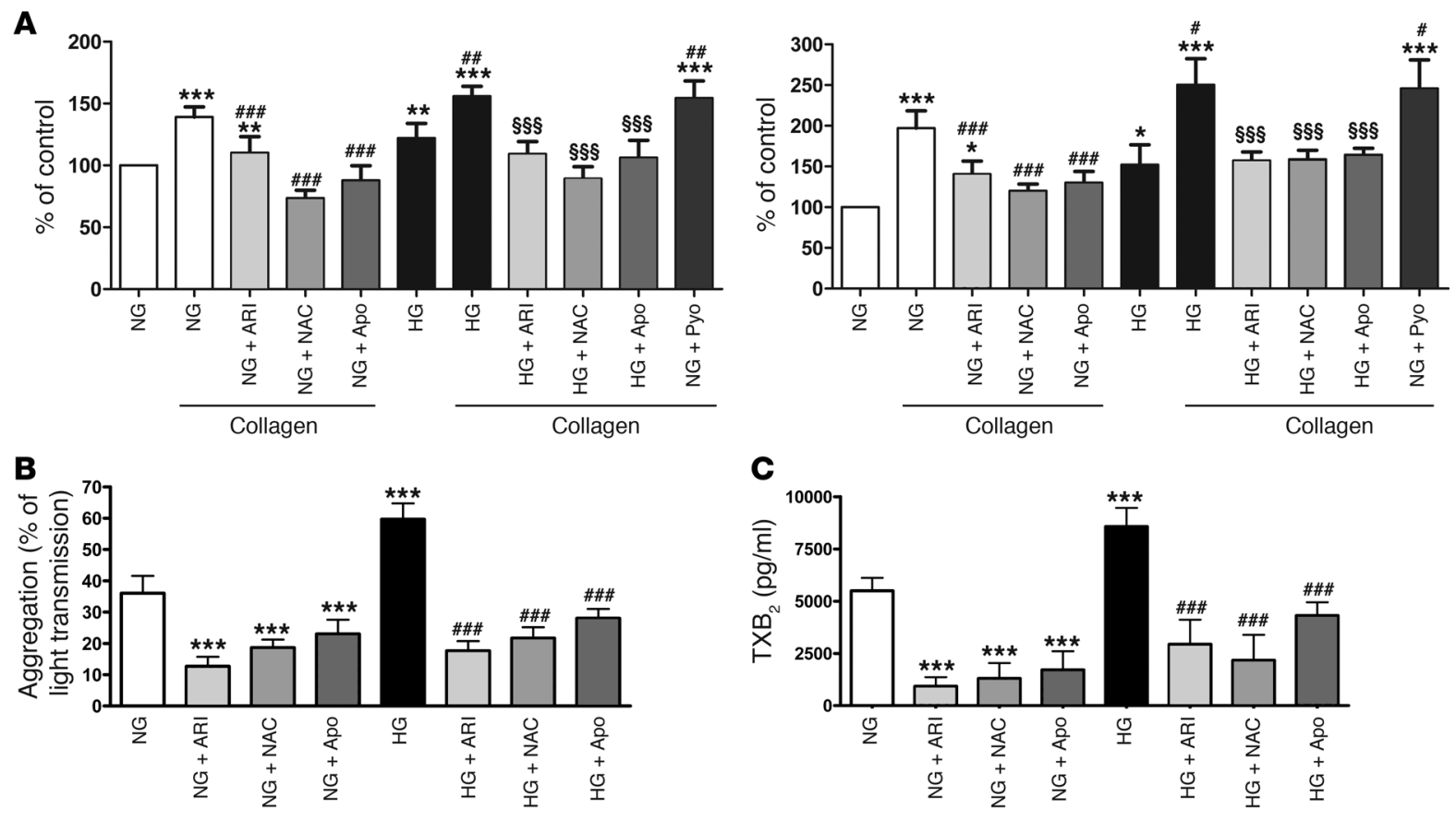

\section{Figure 6}

AR contributes to oxidative stress in collagen-stimulated platelets. Platelet suspensions were incubated with NG or HG for 90 minutes in the presence or absence of $10 \mu \mathrm{mol} / /$ epalrestat, $1 \mathrm{mmol} / \mathrm{I} \mathrm{NAC}$, and $100 \mu \mathrm{mol} / \mathrm{l}$ apocynin (Apo). (A) Washed platelets were incubated with $1 \mu \mathrm{mol} / \mathrm{I}$ ROS/Superoxide Detection Mix for 60 minutes at $37^{\circ} \mathrm{C}$ in the presence or absence of $1 \mu \mathrm{g} / \mathrm{ml}$ collagen for 10 minutes. The quantification of data for ROS is shown in the left panel, and that for superoxide is shown in the right panel. Data are expressed as mean $\pm \mathrm{SD}(n=5 \mathrm{HS}){ }^{* * *} P<0.001$, ${ }^{* *} P<0.01$, and ${ }^{*} P<0.05$ compared with values incubated in NG alone; \#\#\# $P<0.001$, \#\# $P<0.01$, and $\#<0.05$ compared with values in NG with the addition of $1 \mu \mathrm{g} / \mathrm{ml}$ collagen; $\$ \S \S P<0.001$ compared with values in $\mathrm{HG}$ with the addition of $1 \mu \mathrm{g} / \mathrm{ml}$ collagen. (B) Aggregation expressed as the percentage of light transmission and measured in platelet suspensions under NG or HG conditions. (C) Levels of TXB ${ }_{2}$ assayed in the supernatant. Data are expressed as mean $\pm \mathrm{SD}(n=5 \mathrm{HS}) .{ }^{* \star *} P<0.001 \mathrm{compared}$ with values incubated in NG with the addition of $1 \mu \mathrm{g} / \mathrm{ml}$ collagen; \#\# $P<0.001$ compared with values in $\mathrm{HG}$ with the addition of $1 \mu \mathrm{g} / \mathrm{ml}$ collagen.

of ASA. The mechanism for this apparent insensitivity to ASA in the presence of thrombosis and DM required further exploration.

ASA ex vivo does not cause a complete saturation of platelet $C O X-1$ activity. In order to verify whether enhanced generation of $\mathrm{TXA}_{2}$ in vivo was a consequence of inadequate inhibition of platelet COX-1 (ex vivo) by ASA administration, we compared residual generation of $\mathrm{TXB}_{2} 12$ hours after the last dose of ASA in whole blood allowed to clot for 1 hour at $37^{\circ} \mathrm{C}$ (serum $\mathrm{TXB}_{2}$ ), which is an index of maximal capacity of platelets to generate $\mathrm{TXB}_{2}$.

We detected enhanced median values of residual generation of platelet $\mathrm{TXB}_{2}$ in $\mathrm{DM}$ patients versus HS; the differences were not statistically significant. However, $12 \%$ of DM patients had higher values of residual $\mathrm{TXB}_{2}$ (i.e., $4 \mathrm{ng} / \mathrm{ml}$ ) than the upper extreme values of serum $\mathrm{TXB}_{2}$ detected in control subjects after ASA administration. In order to verify whether in these patients higher generation of platelet $\mathrm{TXB}_{2}$ was due to the phenomenon of ASA resistance, which implies that the ASA target, i.e., COX-1, is less sensitive to inactivation by ASA, we assessed residual generation of $\mathrm{TXB}_{2}$ after adding ASA $(50 \mu \mathrm{g} / \mathrm{ml})$ in vitro. As shown in Figure 12, in vitro addition of ASA caused a further significant reduction of $\mathrm{TXB}_{2}$ generation in both DM patients and HS. Importantly, only $2 \%$ of patients had residual $\mathrm{TXB}_{2}$ levels higher than $4 \mathrm{ng} / \mathrm{ml}$ in DM groups with ASA in vitro. Our results (Figure 12) show that in almost all DM patients, ASA is able to reduce serum $\mathrm{TXB}_{2}$ to a similar extent when in vivo followed by in vitro ASA treatment of patients' blood is used. Thus, the apparent ASA insensitivity and platelet hyperactivity is, in most cases, not due to ASA resistance, but rather a combination of inadequate ASA bioavailability and increased TX production (and receptor expression) via the AR/ROS pathway. It is important to point out that the observed residual COX-1 activity is present even after ASA treatment. This COX-1 activity likely converts the increased AA substrate generated from the p38 $\alpha \mathrm{MAPK} / \mathrm{PLA}_{2}$ activation pathway, leading to enhanced platelet $\mathrm{TXA}_{2}$ generation and increased platelet activation under conditions of acute hyperglycemia and collagen activation.

Analysis of human TP genetic variants in cardiovascular patients. In order to ensure and confirm that the increase in TX levels was not secondary to a dysfunctional or defective TP, genomic DNA from patients and volunteers from the Yale (human platelet signaling studies), Chieti (study groups 1 and 2), and Johns Hopkins (study group 3) studies was sequenced for variants in the TP gene (TBXA2R). No dysfunctional nonsynonymous mutations could account for the observed results (data not shown). This result demonstrates that the difference in TX-M levels between the DM and non-DM patients could not be attributed to structural defects in the human TP (hTP).

\section{Discussion}

We set out to discover the mechanism leading to TX release and platelet hyperactivity in DM. We demonstrate for what we 
A
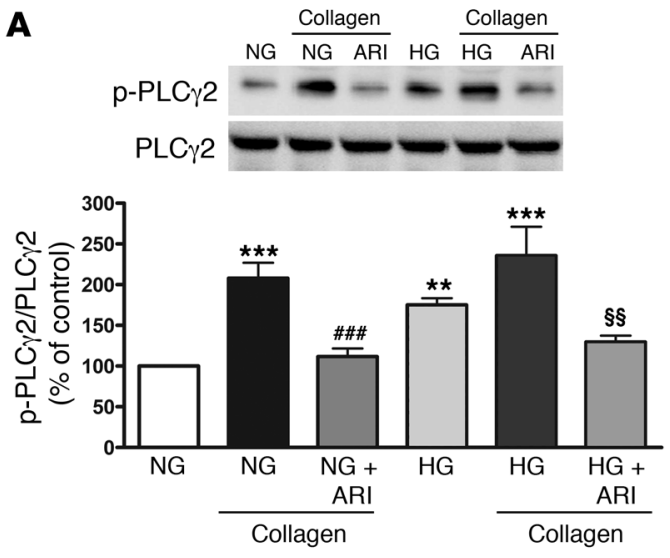

B

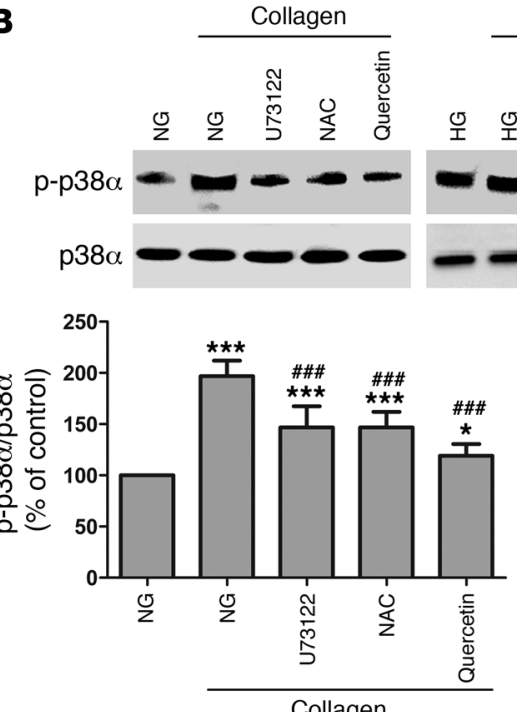

Collagen
Collagen

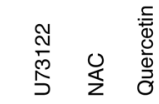

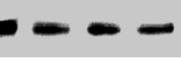
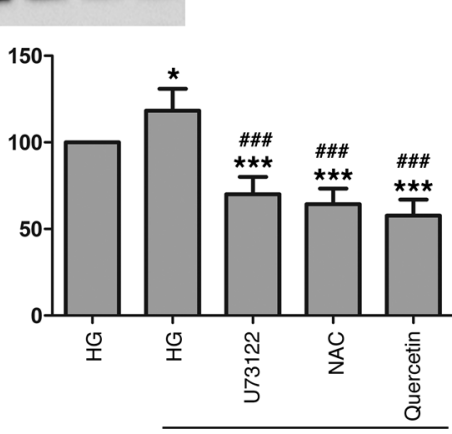

Collagen

\section{Figure 7}

$\mathrm{AR}$ is required for PLC phosphorylation in collagen-stimulated platelets. (A) Platelet suspensions were incubated with $\mathrm{NG}$ or $\mathrm{HG}$ for 90 minutes in the presence or absence of $10 \mu \mathrm{mol} / \mathrm{l}$ epalrestat prior to stimulation by $1 \mu \mathrm{g} / \mathrm{ml}$ collagen for 10 minutes, and the total extract was harvested for experiments. Data are expressed as mean $\pm \operatorname{SD}(n=5$ HS). ${ }^{* * *} P<0.001$ and ${ }^{* *} P<0.01$ compared with values incubated in NG alone; \#\# $P<0.001$ compared with values in NG with the addition of $1 \mu \mathrm{g} / \mathrm{ml}$ collagen; $\$ \S P<0.01$ compared with values in $\mathrm{HG}$ with the addition of $1 \mu \mathrm{g} / \mathrm{ml}$ collagen. p-PLC $\gamma 2$, phospho-PLC $\gamma 2$. (B) Inhibition of the upstream pathway of $\mathrm{p} 38 \alpha$ MAPK attenuated its phosphorylation in collagen-stimulated platelets. Platelet suspensions were incubated with $\mathrm{NG}$ or $\mathrm{HG}$ for 90 minutes in the presence of $5 \mu \mathrm{mol} / / \mathrm{U} 73122,1 \mathrm{mmol} / \mathrm{I} \mathrm{NAC}$, and 10 $\mu \mathrm{mol} / \mathrm{l}$ quercetin prior to stimulation by $1 \mu \mathrm{g} / \mathrm{ml}$ collagen for 10 minutes, and the total extract was harvested for experiments. Data are expressed as mean \pm SD $(n=5 \mathrm{HS})$. ${ }^{* * *} P<0.001$ and ${ }^{*} P<0.05$ compared with values incubated in NG or HG alone, respectively; \#\#\# $P 0.001$ compared with values in NG or $\mathrm{HG}$ with the addition of $1 \mu \mathrm{g} / \mathrm{ml}$ collagen, respectively. p-p38 $\alpha$, phospho-p38 $\alpha$. believe is the first time that AR plays a central role in synergistically transducing hyperglycemia and collagen exposure to TX release, increased cell surface TP expression, and enhanced platelet activity. This effect is associated with ROS generation and the activation of PLC $\gamma 2$, PKC $\beta$ II, PKC $\delta$, and p38 $\alpha$ MAPK (Figure 13). Our clinical studies support these results, as increased $\mathrm{TXA}_{2}$ generation in vivo, particularly in DM patients, reflected underlying thrombovascular disease (DVT and CABG thrombosis). These mechanistic insights may explain in part the poor prognosis observed with patients presenting with hyperglycemia and acute coronary syndrome (24-30). Enhanced platelet activity due to a combination of hyperglycemia and collagen exposure may predispose to further thromboembolic disease.

Complex regulatory pathway required for the synergistic effects of glucose on collagen-stimulated aggregation. The enhanced utilization of the AR pathway during hyperglycemia is well known to contribute to disease in many tissues, including microvascular and macrovascular complications, polyneuropathy, and retinopathy in DM patients $(10,11)$. Surprisingly, its role and relationship to TX release and platelet hyperactivity has been unexplored. We now demonstrate that AR plays a critical role in transducing collagen signaling in platelet aggregation and that this is further enhanced in conditions of hyperglycemia. Our mechanistic studies are supported by a recent study using $2 \mathrm{D}$ difference gel electrophoresis and mass spectrometry, which demonstrated that the expression of AR was significantly increased in the activated human platelets after stimulation of the GPVI receptor (14). The binding of collagen to GPVI results in receptor clustering and phosphorylation of the tyrosine kinase Syk and the activation of PLC (31). In platelets, many PLC isoforms have been identified, and evidence suggests that the signaling through collagen-induced aggregation is mediated by the receptor-bound tyrosine kinases and activation of PLC $\gamma(32,33)$. We have now demonstrated that PLC $\gamma 2$ phosphorylation is greatly increased in the glucose-treated platelets and collagen-induced platelet aggregation and that PLC inhibition using U73122 effectively prevented collagen-induced $\mathrm{TXB}_{2}$ generation and aggregation, suggesting that PLC $\gamma 2$ phosphorylation is required for collagen-induced platelet aggregation. Looking further downstream (Figure 13), in HG- and collagen-stimulated platelets, PKC $\alpha, \mathrm{PKC} \beta \mathrm{II}$, and PKC $\delta$ were translocated from cytosol to membrane, with concomitant increase in their phosphorylated forms, thus supporting a role of PKC $\alpha, \mathrm{PKC} \beta \mathrm{II}$, and PKC $\delta$ in this process. These findings are also supported by previous studies that demonstrate PKC $\alpha, \mathrm{PKC} \beta \mathrm{II}$, and PKC $\mathrm{P}$ as playing a crucial role in collagen-induced platelet aggregation $(21,22)$. Thus, a complex web of signaling components is involved in platelet activation upon exposure to collagen and hyperglycemia (Figure 13). Our study unifies many of the platelet-dependent signaling compo- 


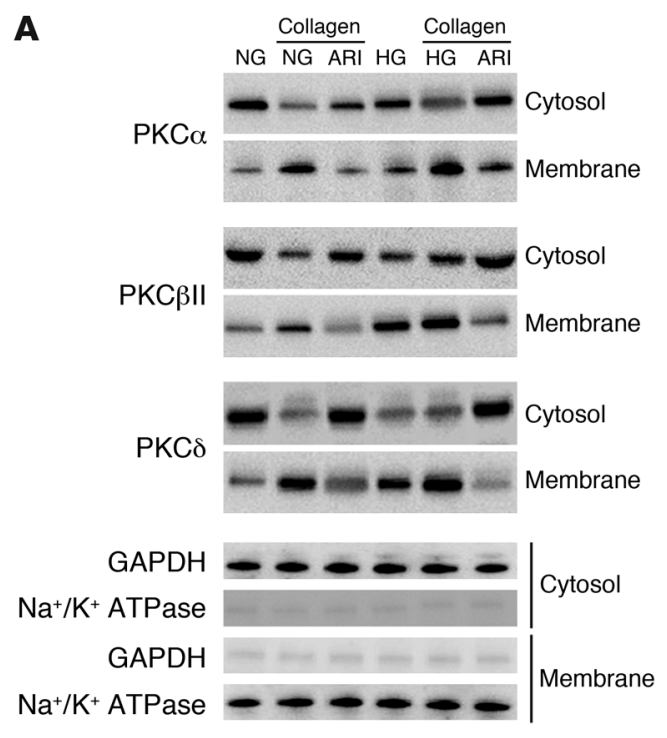

Figure 8

AR is required for the intracellular translocation of PKC isoforms $(\alpha$, $\beta \mathrm{II}$, and $\delta$ ) in collagen-stimulated platelets. (A) Platelet suspensions were incubated with NG or HG for 90 minutes in the presence or absence of $10 \mu \mathrm{mol} / \mathrm{l}$ epalrestat prior to stimulation by $1 \mu \mathrm{g} / \mathrm{ml}$ collagen for 10 minutes, and the membrane and cytosolic fractions were harvested for Western blot. Representative blots are shown. Expressions of $\mathrm{PKC}$ isoforms in cytosolic and membrane fractions were normalized to GAPDH and $\mathrm{Na}^{+} / \mathrm{K}^{+}$ATPase expression, respectively. Quantitative results of (B) PKC $\alpha,(\mathbf{C}) \mathrm{PKC} \beta \mathrm{II}$, and (D) PKC $\delta$ expression were plotted in histograms. Data are expressed as mean \pm SD $(n=5 \mathrm{HS}) .{ }^{* * *} P<0.001$ compared with values incubated in NG alone; $\# \# P<0.001$ and ${ }^{\#} P<0.01$ compared with values in NG with the addition of $1 \mu \mathrm{g} / \mathrm{ml}$ collagen; $\$ \S \S P<0.001$ and $\$ \S P<0.01$ compared with values in $\mathrm{HG}$ with the addition of $1 \mu \mathrm{g} / \mathrm{ml}$ collagen.

nents in the AR transduction of glucose signaling. AR appears to serve as a master switch that causes collagen-induced platelet aggregation to be enhanced by the presence of HG.

Oxidative stress plays an important role in TX-induced platelet byperactivity. Glucose flux through AR is well recognized to generate oxidative stress by several mechanisms, including NADPH depletion, decreasing glutathione (GSH) levels (NADPH is the cofactor for GSH reductase that regenerates GSH from GSH disulfide [GSSG]), and increasing advanced glycation end products, generating ROS (10). Previous studies have demonstrated that TXA 2 biosynthesis is upregulated by oxidant formation (e.g., hydrogen peroxide) during platelet aggregation $(34-36)$. We and others $(16,20)$ have demonstrated that hyperglycemia increases oxidative stress in platelets. We show that this potentiates $\mathrm{p} 38 \alpha \mathrm{MAPK} / \mathrm{CPLA}_{2}$ signaling, which catalyzes AA release, producing $\mathrm{TXA}_{2}$. Interestingly, ROS derived from platelet activation also plays an important role in signaling upon collagen-induced platelet aggregation. Free radical species act as secondary messengers that increase cytosolic $\mathrm{Ca}^{2+}$ during the initial phase of platelet activation processes, and PKC is involved in receptor-mediated ROS production in platelets (36). Previous
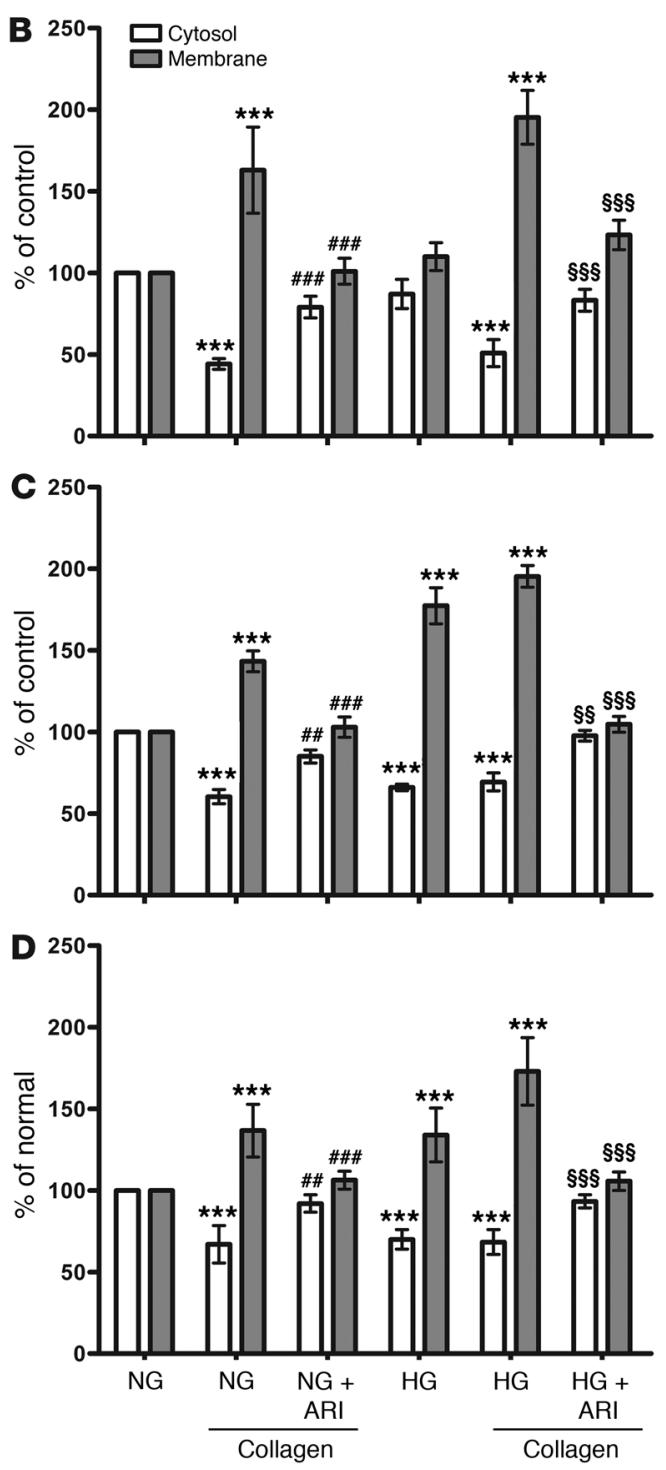

studies also showed that ROS formation is involved in collageninduced platelet aggregation, which is dependent on AA release and its metabolism (34); thus, ROS may be needed for platelet activation (37). Indeed, our studies demonstrate that oxidative stress is significantly increased in collagen-stimulated platelets, and inhibition of AR partly attenuated collagen-induced oxidative stress, supporting an important role for AR-exacerbated oxidative stress in collagen-dependent signaling. Furthermore, our current study and those of others have now demonstrated oxidative stress-induced stabilization and relocation of TP to the cell surface $(38,39)$.

Positive feedback cycle of $T X$ release and platelet hyperactivity. Urinary TX-M, reflecting the whole biosynthesis of TXA ${ }_{2}$ in the body, mainly by platelets with a minor contribution from extraplatelet sources, is significantly higher in DM, with the absolute post-ASA values in DM patients being comparable to those of controls who did not receive ASA $(7,8)$. Many believe enhanced $\mathrm{TXA}_{2}$ biosynthesis in vivo in T2DM is predominantly derived from platelets (7), and such studies support our findings that platelet degranulation and synthesis of $\mathrm{TXA}_{2}$ mediate further platelet activation in DM. This then raises the issue of identification of the initiating events that 
A

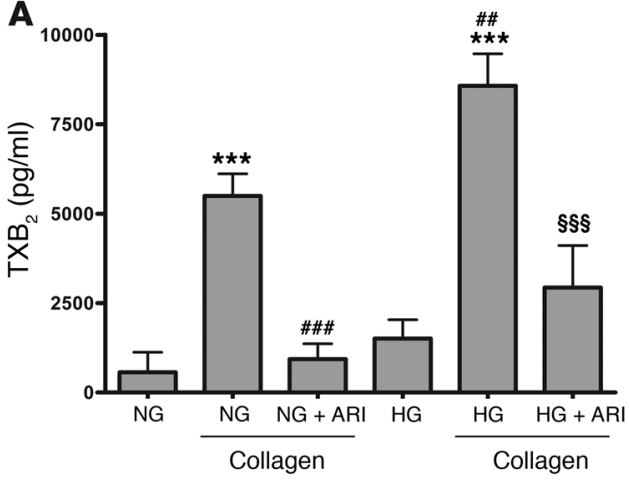

C

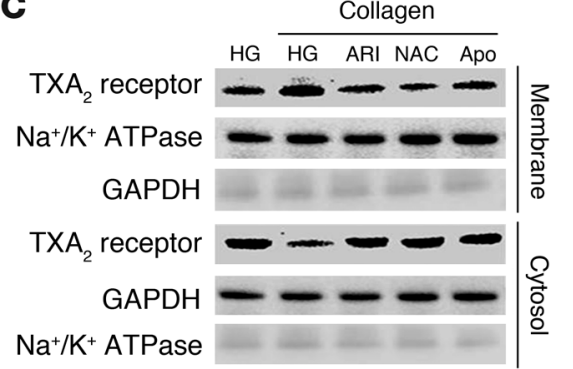

B
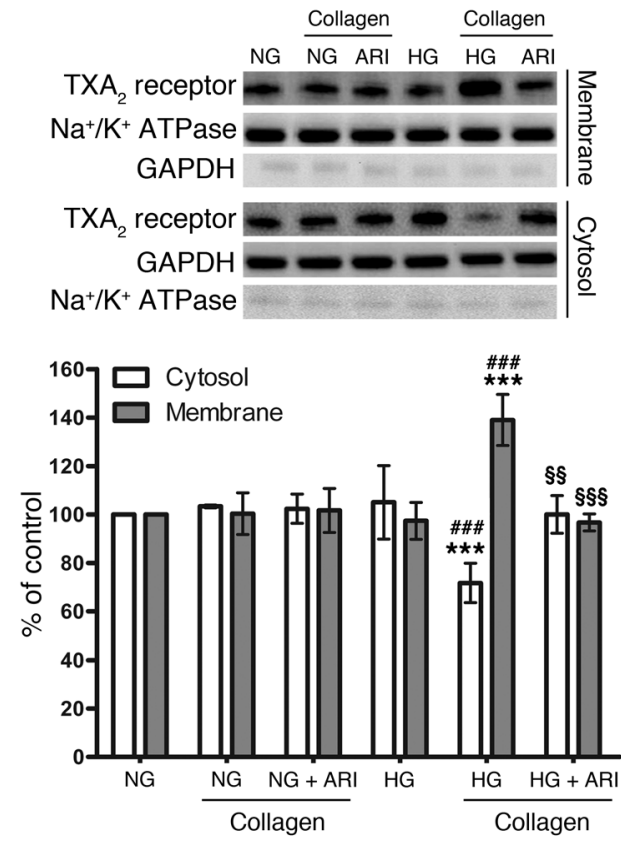

Figure 9

$A R$ is involved in TX signaling in platelets. Role of AR in (A) TX generation and (B) surface expression of TP in collagen-stimulated platelets. Platelet suspensions were incubated with NG or $\mathrm{HG}$ for 90 minutes in the presence or absence of $10 \mu \mathrm{mol} / \mathrm{l}$ epalrestat prior to stimulation with $1 \mu \mathrm{g} / \mathrm{ml}$ collagen for 10 minutes. Levels of $\mathrm{TXB}_{2}$ were assayed in the supernatant. Expression of TP was assessed in the membrane and cytosolic fractions. Expression of TP in cytosolic and membrane fraction was normalized to GAPDH and $\mathrm{Na}^{+} / \mathrm{K}^{+}$ ATPase expression, respectively. Data are expressed as mean \pm SD $(n=5 \mathrm{HS}) .{ }^{* * *} P<0.001 \mathrm{com}-$ pared with values incubated in NG alone; \#\# $P<0.001$ and \#\# $P<0.01$ compared with values in NG with the addition of $1 \mu \mathrm{g} / \mathrm{ml}$ collagen; $\S \S \S P<0.001$ and $\S \S P<0.01 \mathrm{com}-$ pared with values in $\mathrm{HG}$ with the addition of $1 \mu \mathrm{g} / \mathrm{ml}$ collagen. (C) Expression of TP was assessed in the membrane and cytosolic fractions. Data are expressed as mean $\pm \mathrm{SD}(n=5 \mathrm{HS}) .{ }^{* * *} P<0.001 \mathrm{com}-$ pared with values incubated in $\mathrm{HG}$ alone; \#\#\# $P<0.001$ compared with values in $\mathrm{HG}$ with the addition of $1 \mu \mathrm{g} / \mathrm{ml}$ collagen. lead to this cascade of hyperactivation, thrombosis, and hyperactivation. As described, it may be that the trigger is endothelial damage (collagen exposure) and that, when combined with hyperglycemia-induced oxidative stress, ROS initiates an amplifying cycle of increased PLC/PKC/p38 $\alpha$ MAPK signaling, $\mathrm{TXA}_{2}$ generation, and platelet hyperactivation. Interestingly, previous animal studies demonstrated that enhanced platelet aggregation and TX synthesis may be detected within days of triggering DM with streptozotocin, suggesting that alterations in platelets and endothelial damage (with collagen exposure) may occur early in the DM state (8). Extraplatelet factors such as NO and prostacyclin are keys to breaking such a cycle; however, there is growing evidence that these naturally occurring antiaggregatory agents are reduced in DM (40).

As our DM patients were on ASA, it was necessary to determine why TX production remained elevated, particularly in the patients from study group 1 (Figure 1). We offer 2 major reasons based upon our results. First, as described, hyperglycemia synergizes with collagen stimulation in platelets to activate the AR-dependent polyol pathway, which increases oxidative stress. The oxidative stress can increase $\mathrm{TXA}_{2}$ production by potentiating $\mathrm{p} 38 \alpha$ $\mathrm{MAPK} / \mathrm{CPLA}_{2}$ signaling, thus providing more substrates (AA) for uninhibited COX-1 to produce $\mathrm{TXA}_{2}$. Additionally, hyperglycemia- and collagen-induced oxidative stress from the AR-dependent polyol pathway can also lead to enhanced expression of TP, increasing the potency of any circulating $\mathrm{TXA}_{2}$. When combined with reduced bioavailability, as observed with the $100 \mathrm{mg}$ (enteric coated) daily usage patients, and thus incomplete inhibition of COX-1 (Figure 12), the combination results in a marked increase in $\mathrm{TXA}_{2}$ over that in control patients (Figure 1). Such differences are less marked when patients are on $325 \mathrm{mg}$ ASA (study group 3, Reduction in Graft Occlusion Rates [RIGOR] group; Figure 11B). Thus AR-dependent signaling pathways upon glucose/collagen platelet activation may provide, in part, an explanation for apparent ASA insensitivity in DM patients and may shed light on the concept of ASA insensitivity in general.

$A R$ inhibitors as antiplatelet therapy? Our study highlights the importance of concurrent clinical studies to substantiate in vitro findings. DM patients with high urinary levels of TX-M despite ASA treatment may have underlying occult endothelial damage and thrombovascular disease. We propose that in the context of vascular damage with collagen exposure and HG, unacetylated platelet COX-1 despite ASA may participate in $\mathrm{TXA}_{2}$ biosynthesis on a background of increased p $38 \alpha$ MAPK-induced PLA $A_{2}$ activity and thus enhanced AA substrate. Higher doses of ASA or lowdose ASA given twice daily may translate into an appropriate suppression of TXA 2 in vivo (assessing the levels of urinary TX-M). Alternatively, the addition of AR inhibitors should be considered to mitigate enhanced $\mathrm{TXA}_{2}$ generation despite ASA treatment in 

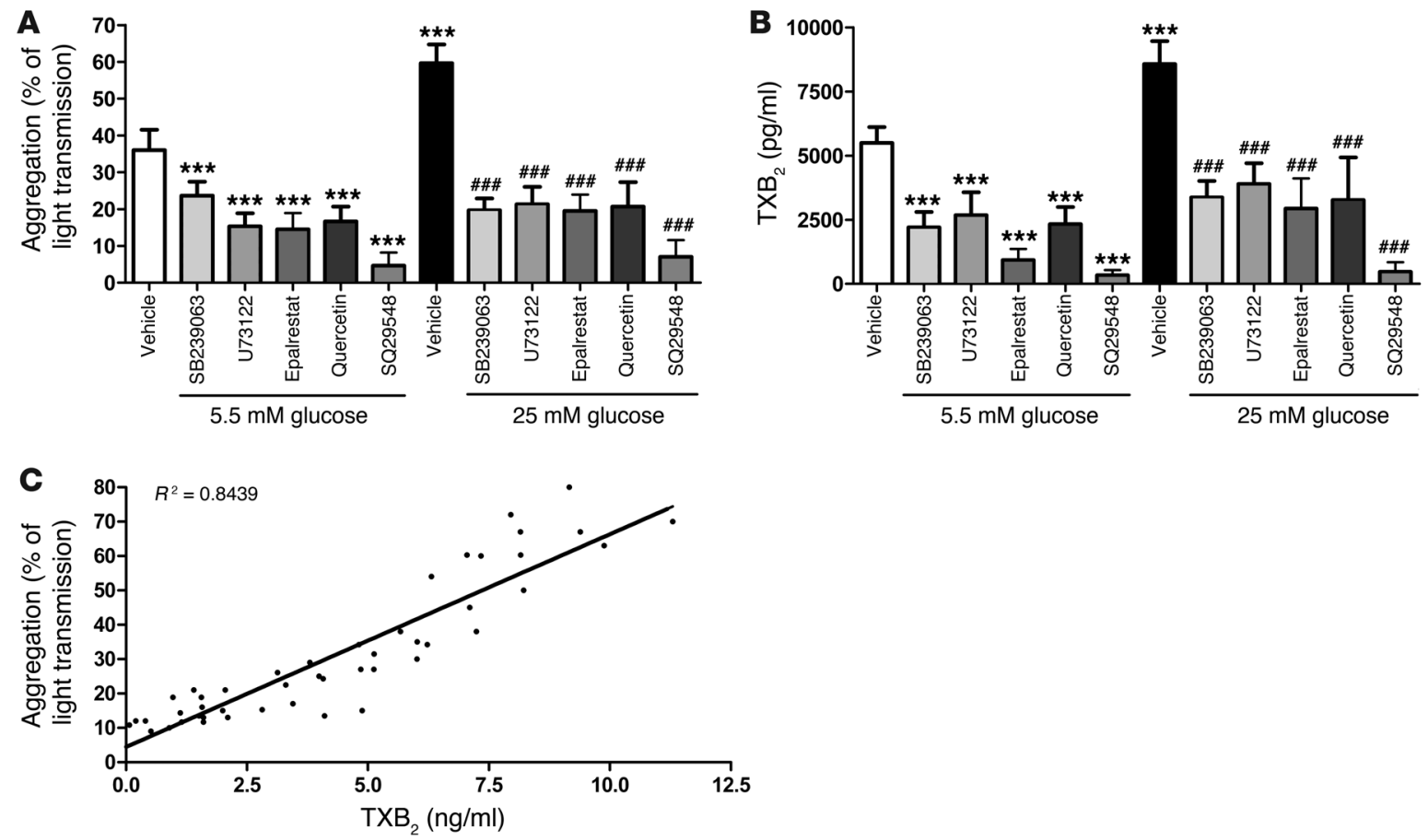

Figure 10

Activation of p38 $\alpha$ MAPK signaling pathway is required for TX generation and aggregation in collagen-stimulated platelets. Platelet suspensions

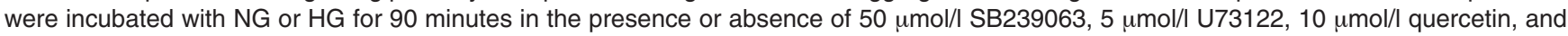
$10 \mu \mathrm{mol} / \mathrm{I} \mathrm{SQ29548} \mathrm{prior} \mathrm{to} \mathrm{stimulation} \mathrm{by} 1 \mu \mathrm{g} / \mathrm{ml}$ collagen. (A) The platelet aggregation was presented as the percentage of light transmission and measured in platelet suspensions under NG or HG condition in response to $1 \mu \mathrm{g} / \mathrm{ml}$ collagen for 5 minutes. (B) After stimulation, the supernatant was harvested for the measurement of $\mathrm{TXB}_{2}$ generation. Data are expressed as mean $\pm \mathrm{SD}(n=5 \mathrm{HS})$. ${ }^{* \star} P<0.001$ compared with vehicle incubated in NG; \#\# $P<0.001$ compared with vehicle incubated in HG. (C) Correlation of TX release with increased platelet aggregation in collagen-stimulated human platelets. Platelet suspensions were incubated with NG or HG for 90 minutes prior to stimulation by $1 \mu \mathrm{g} / \mathrm{ml}$ collagen for $10 \mathrm{minutes}$; the percentage of light transmission was also measured in platelet suspensions, and the TX release was assayed in the supernatant.

DM patients. Epalrestat used in the present study is an AR inhibitor that is approved in Japan to prevent or slow the progression of DM neuropathy $(41,42)$. Our study using human platelets demonstrates that hyperglycemia-induced TX signaling and hyperaggregability were effectively attenuated by treatment with epalrestat. The use of AR inhibitors may be particularly effective against postprandial hyperglycemia, which is associated with enhanced lipid peroxidation and platelet activation in early T2DM (43) and increased platelet aggregation in DM patients with microangiopathy (44). The addition of AR inhibitors may be a new therapeutic option to prevent thrombotic events in DM patients.

Limitations of the present studies. There are a number of limitations to our current studies. Foremost, the sizes of our studies are relatively small. Further larger studies assessing both arterial and venous thrombosis in the setting of hyperglycemia and DM are required to corroborate our results. Although we have focused on the platelet-dependent mechanism, this is a reductionist approach, as many other factors from blood cells, the endothelium, and vascular smooth muscle cells clearly play an important role in the development of the DM platelet response. Our studies also focus on acute increases in glucose without addressing the effects of chronic increases. Moreover, there are many other important components to the DM response (e.g., protein glycation) besides AR that likely contribute to increased platelet activity. The final picture is likely to be far more complex, as not all studies in DM patients have demonstrated consistent increases in TX production (45). Despite these limitations, our study provides strong evidence for a multitargeted approach and the foundation and justification for further clinical therapeutic trials to prevent atherothrombotic disease arising from platelet hyperactivity in patients with DM.

Summary. It is currently a subject of debate whether enhanced platelet activity associated with DM is a cause (thrombosis, e.g., plaque rupture) or consequence (metabolic disturbance) of disease. Our studies indicate that both of these are true. The combination of hyperglycemia during collagen activation leads to a positive feedback cycle of release of platelet TX and enhanced platelet aggregation (cause and consequence). Moreover, we demonstrate that AR leading to enhanced ROS production and TX generation plays a major role in this synergistic response. Our findings support the use of higher doses of ASA; however, inhibition of vascular prostacyclin biosynthesis makes this choice inadvisable. The foundation for antiplatelet therapy in DM patients may very well be a combination of ASA with AR inhibitors (and/or TX inhibitors) for both primary and secondary prevention of cardiovascular events.

\section{Methods}

Drugs and inhibitors. The AR inhibitor epalrestat and the NADPH oxidase inhibitor apocynin were purchased from Santa Cruz Biotechnology Inc. The antioxidant NAC was from Fisher Scientific. The p38 MAPK inhibitor SB239063 and the phospholipase C inhibitor U73122 were from Calbio- 
A

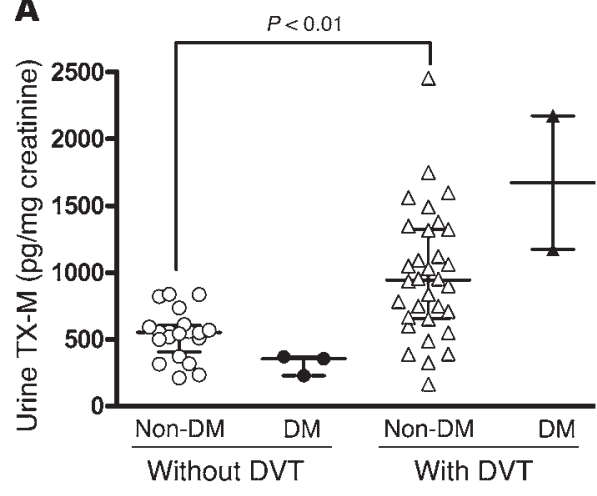

B

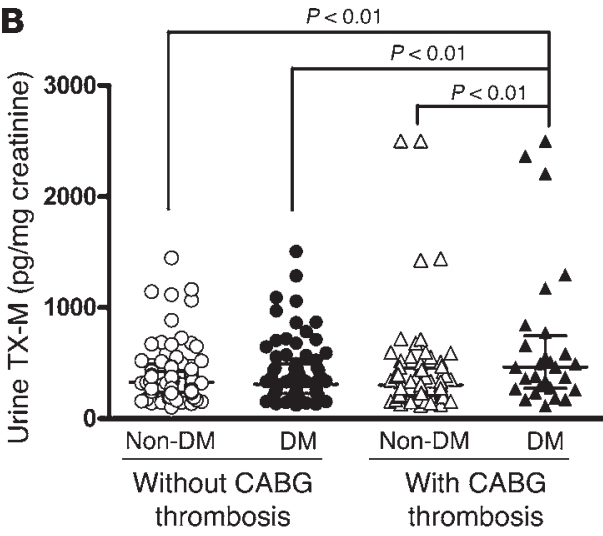

\section{Figure 11}

Increased urinary TX metabolites in patients with DVT or CABG thrombosis. (A) For patients with DVT, the level of urinary TX metabolite was measured as an index of TX release in DM patients with or without DVT ( $n=2$ and $n=3$, respectively) compared with non-DM patients with or without DVT ( $n=32$ and $n=20$, respectively). None of the patients were on ASA therapy. (B) For patients with CABG thrombosis (RIGOR patients), the level of urinary TX metabolite was measured in DM patients with or without CABG thrombosis ( $n=28$ and $n=75$, respectively) compared with non-DM patients with or without CABG thrombosis ( $n=68$ and $n=120$, respectively). All the patients were treated with ASA (325 mg once daily) upon discharge from surgery as described in Methods. Data are expressed as median with IQR.

chem. The TP antagonist SQ29548 was from Cayman Chemical. ASA and another AR inhibitor, quercetin, were from MP Biomedicals.

Preparation of human platelets. Venous blood was drawn from volunteers at Yale University Medical School, who were free from medication known to interfere with platelet function. Platelet-rich plasma (PRP) was prepared from blood $(27 \mathrm{ml})$ that was drawn by venipuncture into $3 \mathrm{ml}$ of $3.8 \%$ trisodium citrate $(\mathrm{w} / \mathrm{v})$. PRP was obtained by centrifugation of blood at $250 \mathrm{~g}$ at $25^{\circ} \mathrm{C}$ for 15 minutes, and the platelet count in the PRP was estimated by an automated cell counter. Platelet-poor plasma (PPP) was obtained by centrifugation of the rest of the blood at $1400 \mathrm{~g}$ at $25^{\circ} \mathrm{C}$ for 10 minutes. The PRP was adjusted with PPP to $2-3 \times 10^{8}$ platelets $/ \mathrm{ml}$ suspension. For the washed platelets, PRP was obtained by centrifugation at $250 \mathrm{~g}$ for 15 minutes, and platelets were sedimented at $1000 \mathrm{~g}$ for 15 minutes and then resuspended in washing buffer $(103 \mathrm{mmol} / \mathrm{l} \mathrm{NaCl}, 5 \mathrm{mmol} / \mathrm{KCl}$, $1 \mathrm{mmol} / 1 \mathrm{MgCl}_{2}, 5 \mathrm{mmol} / \mathrm{l}$ glucose, $36 \mathrm{mmol} / \mathrm{l}$ citric acid, $\mathrm{pH}$ 6.5) containing $3.5 \mathrm{mg} / \mathrm{ml} \mathrm{BSA}$ (Sigma-Aldrich). After sedimentation, the platelets were washed twice in this buffer and resuspended at $2-3 \times 10^{8}$ platelets $/ \mathrm{ml}$ in the buffer composed of $5 \mathrm{mmol} / \mathrm{l}$ HEPES, $137 \mathrm{mmol} / \mathrm{l} \mathrm{NaCl}, 2 \mathrm{mmol} / \mathrm{l}$ $\mathrm{KCl}, 1 \mathrm{mmol} / 1 \mathrm{MgCl}_{2}, 12 \mathrm{mmol} / \mathrm{l} \mathrm{NaHCO} 3,0.3 \mathrm{mmol} / 1 \mathrm{NaH}_{2} \mathrm{PO}_{4}$, and $5.5 \mathrm{mmol} / \mathrm{l}$ glucose, pH 7.4, containing $3.5 \mathrm{mg} / \mathrm{ml} \mathrm{BSA} \mathrm{(46).}$

Measurement of platelet aggregation. Platelet suspensions were incubated with 5.5 or $25 \mathrm{mmol} / \mathrm{l}$ glucose in the presence or absence of 1 or $10 \mu \mathrm{mol} / \mathrm{l}$ epalrestat for 90 minutes at $37^{\circ} \mathrm{C} .5 .5 \mathrm{mmol} / 1$ glucose is a physiological plasma glucose level, whereas $25 \mathrm{mmol} / \mathrm{l}$ glucose is a pathophysiological high level observed in poorly controlled DM patients (47). Platelet aggregation was monitored at $37^{\circ} \mathrm{C}$ with constant stirring $(1200 \mathrm{rpm})$ in a dualchannel lumi-aggregometer (model 700; Chrono-Log). Platelet aggregation was measured as the increase in light transmission for 10 minutes, starting with the addition of $0.5 \mu \mathrm{lof} 1 \mathrm{mg} / \mathrm{ml}$ collagen (Chrono-Log) into $500 \mu \mathrm{l}$ as a proaggregatory stimulus; the final concentration was $1 \mu \mathrm{g} / \mathrm{ml}$. For measuring ADP-stimulated platelet aggregation, $1 \mu \mathrm{M}$ ADP (Chrono-Log) was used as the final concentration. The maximum aggregation was expressed as a percentage of maximum light transmission, with nonstimulated PRP being $0 \%$ and PPP $100 \%$.

Determination of platelet activation. P selectin translocation was assessed by flow cytometry using monoclonal antibody against $\mathrm{P}$ selectin (fluorescein isothiocyanate-conjugated, AK4; Biolegend), as described previously (48), with minor modifications. Briefly, platelet suspensions were preincubated with 5 or $25 \mathrm{mmol} / \mathrm{l}$ glucose in the presence or absence of 1 or $10 \mu \mathrm{mol} / 1$ epalrestat for 90 minutes prior to stimulation by $1 \mu \mathrm{g} / \mathrm{ml}$ collagen for 10 minutes. The samples were then washed and fixed in $1 \%$ paraformaldehyde for 1 hour at $4{ }^{\circ} \mathrm{C}$. The fixed platelets were then washed and labeled with saturating concentrations of monoclonal antibody for 1 hour. All the samples were analyzed on a flow cytometer (FACSCalibur). Platelets were identified and gated by their characteristic forward- and side-scatter properties, and 20,000 platelets were analyzed from each sample.

Western blotting. Platelets were homogenized in the lysis buffer $(25 \mathrm{mmol} / 1$ Tris $\mathrm{HCl}, \mathrm{pH} 7.6,150 \mathrm{mmol} / \mathrm{l} \mathrm{NaCl}, 1 \% \mathrm{NP}-40,1 \%$ sodium deoxycholate, $0.1 \%$ SDS), with the addition of $0.1 \%$ protease inhibitor and $0.1 \%$ phosphatase inhibitor (Thermo Scientific). The supernatant was collected after centrifugation at $15,700 \mathrm{~g}$ for 15 minutes at $4^{\circ} \mathrm{C}$. The samples were loaded in $4 \times$ SDS-Laemmli sample buffers and boiled for 10 minutes. Equal amounts of total extracts were subjected to SDS-PAGE. The proteins were transferred to nitrocellulose membrane and probed with the indicated antibodies, and the antigen-antibody complex was detected by enhanced chemiluminescence reagents (Thermo Scientific). Primary antibodies against the following proteins were diluted and used according to the manufacturer's instructions: AR (sc-17732; Santa Cruz Biotechnology Inc.), PKCa (sc-208; Santa Cruz Biotechnology Inc.) and its phosphorylated form (sc-136018, Thr638; Santa Cruz Biotechnology Inc.),

\section{Table 1}

Cardiovascular risk factors for the populations studied

$\begin{array}{lcc} & \text { DVT study } & \text { RIGOR study } \\ n & 57 & 291 \\ \text { Male/female } & 17 / 40 & 230 / 61 \\ \text { Median age (range) } & 62.5(33-82) & 63(55-72) \\ \text { BMI } & 29.2( \pm 5.7) & 29.5( \pm 6.13) \\ \text { Dyslipidemia } & 34(50.0 \%) & 191(83.4 \%) \\ \text { DM } & 8(11.8 \%) & 84(36.7 \%) \\ \text { Hypertension } & 35(51.5 \%) & 187(81.7 \%) \\ \text { Smokers } & 10(14.7 \%) & 52(22.7 \%)\end{array}$




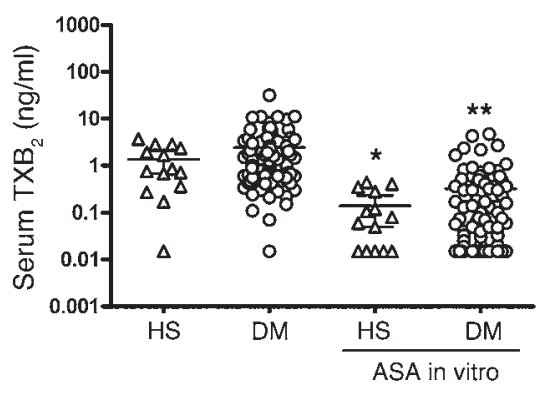

Figure 12

Effect of in vitro administration of $A S A$ on the residual $\mathrm{TXB}_{2}$ generation in HS and DM patients. Serum TXB ${ }_{2}$ levels, markers of platelet COX-1 activity, were assessed in HS $(n=14)$ and DM $(n=102)$ patients with low-dose ASA (100 mg/day). Residual generation of $\mathrm{TXB}_{2}$ was assessed in whole blood allowed to clot for 1 hour at $37^{\circ} \mathrm{C} 12$ hours after the last dose of ASA. Data are expressed as median with IQR. ${ }^{* *} P<0.01$ and ${ }^{*} P<0.05$ compared with values in the corresponding group without ASA in vitro.

PKC $\beta$ II (NB110-57358; Novus Biologicals) and its phosphorylated form (NSB964, Thr641; Novus Biologicals), PKCס (2058; Cell Signaling Technology) and its phosphorylated form (9376, Ser643; Cell Signaling Technology), PLC $\gamma 2$ (NB110-66669; Novus Biologicals) and its phosphorylated form (NB110-57424, Tyr1217; Novus Biologicals), p38 $\alpha$ MAPK (9212; Cell Signaling Technology) and its phosphorylated form (AF869, T180/Y182;
R\&D Systems), and TP (NBP1-40198; Novus Biologicals). Secondary antibodies against rabbit and mouse $\operatorname{IgG}$ (Thermo Scientific) were diluted to 1:5000 before use. The membrane was reprobed with antibodies against $\mathrm{Na}^{+} / \mathrm{K}^{+}$-ATPase (NB300-147; Novus Biologicals), a plasma membrane marker, and GAPDH (sc-20357; Santa Cruz Biotechnology), a cytoplasmic marker, for normalization.

Measurement of AR activity. The platelets were homogenized and centrifuged as for Western blot analysis. To determine the AR activity, a spectrophotometry measurement was used as described previously (49).

Human phosphokinase array. The human phosphokinase array was performed according to the manufacturer's instructions (R\&D Systems). Briefly, the lysates were diluted to $200 \mu \mathrm{g} / \mathrm{ml}$ using the specific array buffer, mixed with a cocktail of biotinylated detection antibodies, and incubated overnight with nitrocellulose membranes spotted with capture and control antibodies. The signals for the amount of phosphorylated proteins bound were measured by the chemiluminescent detection method.

Subcellular fractionation. Platelets were sedimented by centrifugation at $2500 \mathrm{~g}$ for 5 minutes. The membrane and cytosolic fractions of total extracts were separated using the subcellular protein fractionation kit (Thermo Scientific). Briefly, the platelets were incubated with cytoplasmic extraction buffer for 10 minutes at $4{ }^{\circ} \mathrm{C}$ and then centrifuged at $500 \mathrm{~g}$ for 5 minutes. The supernatant was harvested as the cytoplasmic extract. The pellet was reconstituted with the ice-cold membrane extraction buffer and incubated for 10 minutes at $4^{\circ} \mathrm{C}$. After incubation, it was then centrifuged at $3000 \mathrm{~g}$ for 5 minutes, and the supernatant was harvested as the membrane extract. Aliquots (60-100 $\mu$ g protein) of the soluble and the membrane fractions were separated by SDS-PAGE and subjected to Western blot

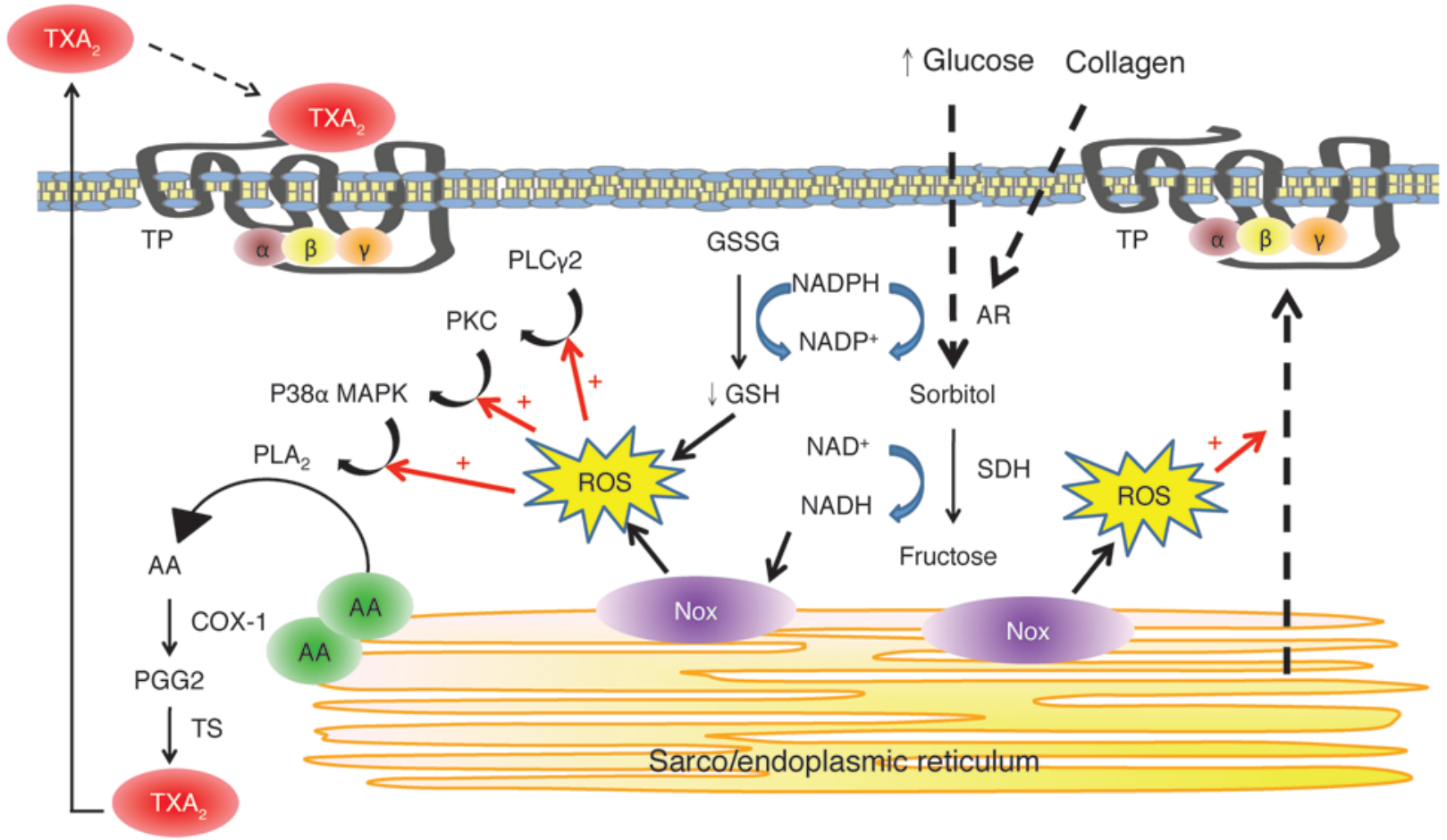

\section{Figure 13}

Proposed mechanism for hyperglycemia-induced platelet hyperactivity through TX signaling mediated by the AR pathway. Hyperglycemia synergizes with collagen stimulation in platelets to activate the AR-dependent polyol pathway, leading to increased ROS production (via GSH depletion and NADH oxidase [Nox]). Excessive ROS can increase $\mathrm{TXA}_{2}$ production by potentiating PLC $\gamma 2 / \mathrm{PKC} / \mathrm{p} 38 \alpha \mathrm{MAPK}$ signaling, thus providing more arachidonic acid substrate for COX-1 and TXA 2 synthase (TS) to produce $\mathrm{TXA}_{2}$, leading to platelet hyperactivity. The oxidative stress can also lead to enhanced surface expression of TP, which increases the potency of circulating $\mathrm{TXA}_{2}$. 
analysis. The blots probed with the antibodies against GAPDH and $\mathrm{Na}^{+} / \mathrm{K}^{+}$ ATPase antibodies were performed to assess the purity of the membrane and cytoplasmic fractions.

Measurement of TX biosynthesis. Platelets were sedimented by centrifugation at $2500 \mathrm{~g}$ for 5 minutes, and the supernatant was harvested for the measurement of TX levels. Since $\mathrm{TXA}_{2}$ is rapidly hydrolyzed into $\mathrm{TXB}_{2}$, the levels of $\mathrm{TXB}_{2}$ were measured using the $\mathrm{TXB}_{2}$ EIA kit according to the manufacturer's ELISA instructions (Cayman Chemical).

Measurement of oxidative stress and superoxide level. The oxidative stress and superoxide levels were measured by a commercial kit according to the manufacturer's instructions (Total ROS/Superoxide Detection Kit; Enzo Life Sciences). Platelets were sedimented by centrifugation at $400 \mathrm{~g}$ for 5 minutes and then washed. The washed platelets were incubated with $1 \mu \mathrm{mol} / \mathrm{l} \mathrm{ROS} /$ Superoxide Detection Mix for 60 minutes at $37^{\circ} \mathrm{C}$ in the presence or absence of $1 \mu \mathrm{g} / \mathrm{ml}$ collagen. Changes in fluorescence intensity were measured using a microplate fluorescence reader (BioTek) at excitation/emission wavelengths of 488/520 nm (oxidative stress) and 550/610 nm (superoxide).

Genetic knockdown of AR in buman megakaryocytes culture. The megakaryocytes (MEG-01 cells) (CRL-2021; ATCC) were cultured in RPMI medium. Cells were transfected with 0.1 or $0.3 \mathrm{mM}$ AR siRNA (sc-37119; Santa Cruz Biotechnology Inc.) using electroporation kits (Lonza). After 48 hours of incubation with RPMI medium containing $5.5 \mathrm{mM}$ glucose, the P selectin translocation to membrane was assessed by flow cytometry in response to $1 \mu \mathrm{g} / \mathrm{ml}$ collagen.

We planned to pursue in vivo mouse studies to confirm our human platelet studies; however, there was significantly reduced AR in mouse platelets (Supplemental Figure 5) and additional indications that mouse platelet collagen activation is not ASA sensitive $(50,51)$. We therefore elected to directly pursue human clinical studies.

Protocol for initial patient study (study group 1). All protocols were approved by the respective Institutional Review Boards. Patients with T2DM $(n=102)$ were recruited from the Hospital Policlinico G.B. Rossi (Verona, Italy) and San Pio da Pietrelcina Hospital. Exclusion criteria included concurrent cancer, inflammatory disorder, immunological disorder, surgery or major trauma in the previous month, or use of NSAIDs or corticosteroids in the previous month. The DM population was on enteric-coated ASA $(100 \mathrm{mg})$ for at least 7 consecutive days (all were chronically treated with low-dose ASA for cardiovascular event prevention). After recruitment, the patients received a standard oral dose of $100 \mathrm{mg}$ for 7 days at 8:00 $\mathrm{pm}$. Timed urine collection (12 hours from 8:00 pm to 8:00 am) was performed on the seventh day of treatment. The HS recruited were from the staff of Hospital Policlinico G.B. Rossi and had normal ECG and echocardiograms and no evidence of atherosclerosis by carotid artery sonography. The HS were all treated with ASA $(100 \mathrm{mg})$ daily also for 7 days. Urine collection (12 hours from 8:00 pm to 8:00 am) was performed on the seventh day of treatment.

Protocol for studies on DVT (study group 2) and saphenous vein graft occlusion (study group 3). For the DVT clinical study (study group 2), all patients with DVT of the lower extremities were diagnosed with duplex ultrasound scanning or peripheral venous angiography. Exclusion criteria were concurrent infection, inflammatory condition or cancer, prolonged immobilization (i.e., lasting more than 7 days) from any cause, recent trauma or surgery (i.e., within the previous 3 months), pregnancy, recent childbirth, or the use of oral contraceptives at the time of the assessment. All patients were not on ASA treatment for at least 2 weeks prior to enrollment. Baseline cardiovascular characteristics were comparable between patients and controls in the DVT subjects, including age, cardiovascular risk factors (cigarette smoking, DM, hypertensive status, and lipid levels), BMI, and systemic inflammatory status index (i.e., CRP and fibrinogen). Urinary TX metabolite TX-M was measured by a previously validated radioimmunoassay technique (52) and expressed as a ratio of urinary creatinine.

The RIGOR study is a multicenter study that investigated the effects of platelet factor 4 and heparin-induced antibody during saphenous vein graft (SVG) occlusion (study group 3). Patients 18 years or older undergoing their first CABG surgery with SVGs were eligible for enrollment. All patients were administered ASA (300 to $325 \mathrm{mg}$ ) 24 hours prior to bypass surgery and at discharge (325 mg ASA, 1 pill daily). Pill counts were performed at each postoperative encounter. SVG patency was assessed by multidetector computed tomography coronary angiography at 6 months. Two blinded reviewers classified the patency with $98 \%$ concordance. A third reviewer adjudicated any discordance. At 6 months, urinary TX-M was also measured along with platelet aggregometry. Details of the original studies and principle findings have been reported in previous studies $(53,54)$. The cardiovascular risk factors for study group 2 (DVT) and study group 3 (RIGOR) are summarized in Table 1.

Statistics. All data were expressed as mean \pm SEM or mean \pm SD where appropriate. One-way ANOVA, followed by Neuman-Keuls multiple comparison test, was used to assess the difference between the mean values of different groups within the same study. For the clinical studies in which results were not a normal distribution, median and IQR (Q1-Q3) were presented graphically. Nonparametric analysis (Mann-Whitney $U$ test) was performed. $P<0.05$ was considered significant.

Study approval. The approval for the human studies was authorized at the respective institutions by the following review boards: study group 1: Ethical Committee of the "Azienda Ospedaliera di Verona," Verona, Italy; study group 2: Comitato di etica per la ricerca biomedica dell'Università degli studi “G. D’Annunzio”; study group 3: Johns Hopkins University Institutional Review Board; ex vivo and in vitro studies: Yale University School of Medicine Human Investigational Committee. All patients provided written informed consent prior to entry into the studies.

\section{Acknowledgments}

This work was supported in part by the NIH (RO1 HL074190 to J. Hwa), an American Heart Association Established Investigator Award (to J. Hwa), the Ministero dell'Istruzione, dell'Università e della Ricerca (PRIN 2006 to P. Patrignani), a Fondazione CarichietiFondazione Negri Sud ONLUS (to V. Evangelista), and by the European Community Sixth Framework Programme (Eicosanox grant LSMH-CT-2004-005033 to P. Patrignani). We also thank Todd Mackenzie (Statistician, Department of Medicine, Dartmouth Medical School) for his statistical advice and analysis, and Stefano Manarini for her technical assistance.

Received for publication June 1, 2011, and accepted in revised form September 7, 2011.

Address correspondence to: John Hwa, Department of Internal Medicine, Section of Cardiovascular Medicine, Yale School of Medicine, Cardiovascular Research Center, 300 George Street, Rm. 759H, New Haven, Connecticut 06511, USA. Phone: 203.737.5583; Fax: 203.737.6118; E-mail: John.Hwa@yale.edu.
1. Beckman JA, Creager MA, Libby P. Diabetes and atherosclerosis: epidemiology, pathophysiology, and management. JAMA. 2002;287(19):2570-2581.

2. Morel O, Kessler L, Ohlmann P, Bareiss P. Diabetes and the platelet: toward new therapeutic para- digms for diabetic atherothrombosis. Atherosclerosis. 2010;212(2):367-376

3. Ferreiro JL, Angiolillo DJ. Diabetes and antiplatelet therapy in acute coronary syndrome. Circulation. 2011;123(7):798-813
4. Carr ME. Diabetes mellitus: a hypercoagulable state. J Diabetes Complications. 2001;15(1):44-54.

5. Bern MM. Platelet functions in diabetes mellitus. Diabetes. 1978;27(3):342-350.

6. Brownlee M. Biochemistry and molecular cell 
biology of diabetic complications. Nature. 2001; 414(6865):813-820.

7. Davi G, et al. Thromboxane biosynthesis and platelet function in type II diabetes mellitus. $N$ Engl J Med. 1990;322(25):1769-1774.

8. Ferroni P, Basili S, Falco A, Davi G. Platelet activation in type 2 diabetes mellitus. J Thromb Haemost. 2004;2(8):1282-1291

9. Bhatnagar A, Srivastava SK. Aldose reductase: congenial and injurious profiles of an enigmatic enzyme. Biochem Med Metab Biol. 1992;48(2):91-121.

10. Chung SS, Ho EC, Lam KS, Chung SK. Contribution of polyol pathway to diabetes-induced oxidative stress. J Am Soc Nephrol. 2003;14(8 suppl 3):S233-S236.

11. Baynes JW, Thorpe SR. Role of oxidative stress in diabetic complications: a new perspective on an old paradigm. Diabetes. 1999;48(1):1-9.

12. Vikramadithyan RK, et al. Human aldose reductase expression accelerates diabetic atherosclerosis in transgenic mice. JClin Invest. 2005;115(9):2434-2443.

13. Marcus AJ, Broekman MJ, Pinsky DJ. COX inhibitors and thromboregulation. N Engl J Med. 2002; 347(13):1025-1026.

14. Schulz C, et al. Identification of novel downstream targets of platelet glycoprotein VI activation by differential proteome analysis: implications for thrombus formation. Blood. 2010;115(20):4102-4110.

15. Kramer RM, et al. p38 mitogen-activated protein kinase phosphorylates cytosolic phospholipase A2 (cPLA2) in thrombin-stimulated platelets. Evidence that proline-directed phosphorylation is not required for mobilization of arachidonic acid by cPLA2. J Biol Chem. 1996;271(44):27723-27729.

16. Coulon L, Calzada C, Moulin P, Vericel E, Lagarde M. Activation of p38 mitogen-activated protein kinase/cytosolic phospholipase A2 cascade in hydroperoxide-stressed platelets. Free Radic Biol Med. 2003; 35(6):616-625.

17. Leoncini G, Bruzzese D, Signorello MG. A role for PLCgamma2 in platelet activation by homocysteine. J Cell Biochem. 2007;100(5):1255-1265.

18. Lee MW, Severson DL. Signal transduction in vascular smooth muscle: diacylglycerol second messengers and PKC action. Am J Physiol. 1994; 267(3 Pt 1):C659-C678

19. Nishizuka Y. Intracellular signaling by hydrolysis of phospholipids and activation of protein kinase C. Science. 1992;258(5082):607-614.

20. Saklatvala J, et al. Role for p38 mitogen-activated protein kinase in platelet aggregation caused by collagen or a thromboxane analogue. J Biol Chem. 1996;271(12):6586-6589.

21. Yacoub D, et al. Essential role of protein kinase C delta in platelet signaling, alpha IIb beta 3 activation, and thromboxane A2 release. J Biol Chem. 2006; 281(40):30024-30035.

22. Gilio K, et al. Functional divergence of platelet protein kinase $\mathrm{C}(\mathrm{PKC})$ isoforms in thrombus formation on collagen. J Biol Chem. 2010;285(30):23410-23419.

23. Saha $P$, et al. Leukocytes and the natural history of deep vein thrombosis: current concepts and future directions. Arterioscler Thromb Vasc Biol. 2011; 31(3):506-512.

24. Deedwania P, et al. Hyperglycemia and acute coronary syndrome: a scientific statement from the American Heart Association Diabetes Committee of the Council on Nutrition, Physical Activity, and Metabolism. Circulation. 2008;117(12):1610-1619.

25 . Kosiborod M, et al. Admission glucose and mortality in elderly patients hospitalized with acute myocardial infarction: implications for patients with and without recognized diabetes. Circulation. 2005;111(23):3078-3086

26 . Foo K, et al. A single serum glucose measurement predicts adverse outcomes across the whole range of acute coronary syndromes. Heart. 2003;89(5):512-516.

27. Iwakura K, et al. Association between hyperglycemia and the no-reflow phenomenon in patients with acute myocardial infarction. J Am Coll Cardiol. 2003;41(1):1-7.

28. Wahab NN, et al. Is blood glucose an independent predictor of mortality in acute myocardial infarction in the thrombolytic era? J Am Coll Cardiol. 2002;40(10):1748-1754

29. Svensson A-M, McGuire DK, Abrahamsson P, Dellborg M. Association between hyper- and hypoglycaemia and 2 year all-cause mortality risk in diabetic patients with acute coronary events. Eur Heart J. 2005;26(13):1255-1261

30. Suleiman M, et al. Fasting glucose is an important independent risk factor for 30-day mortality in patients with acute myocardial infarction: a prospective study. Circulation. 2005;111(6):754-760.

31. Yanaga $F$, et al. Syk interacts with tyrosinephosphorylated proteins in human platelets activated by collagen and cross-linking of the Fc gammaIIA receptor. Biochem J. 1995;311(pt 2):471-478.

32. Rhee SG, Suh PG, Ryu SH, Lee SY. Studies of inositol phospholipid-specific phospholipase C. Science. 1989;244(4904):546-550.

33. Banno Y, Yada Y, Nozawa Y. Purification and characterization of membrane-bound phospholipase C specific for phosphoinositides from human platelets. J Biol Chem. 1988;263(23):11459-11465.

34. Caccese D, et al. Superoxide anion and hydroxyl radical release by collagen-induced platelet aggregation--role of arachidonic acid metabolism. Thromb Haemost. 2000;83(3):485-490.

35. Leo R, et al. Platelet activation by superoxide anion and hydroxyl radicals intrinsically generated by platelets that had undergone anoxia and then reoxygenated. Circulation. 1997;95(4):885-891.

36. Wachowicz B, Olas B, Zbikowska HM, Buczynski A. Generation of reactive oxygen species in blood platelets. Platelets. 2002;13(3):175-182

37. Pignatelli P, Pulcinelli FM, Lenti L, Gazzaniga PP, Violi F. Vitamin E inhibits collagen-induced platelet activation by blunting hydrogen peroxide. Arterioscler Thromb Vasc Biol. 1999;19(10):2542-2547.

38. Wilson SJ, Cavanagh CC, Lesher AM, Frey AJ, Russell SE, Smyth EM. Activation-dependent stabilization of the human thromboxane receptor: role of reactive oxygen species. J Lipid Res. 2009;50(6):1047-1056.

39. Ball SK, Field MC, Tippins JR. Regulation of thromboxane receptor signaling at multiple levels by oxidative stress-induced stabilization, relocation and enhanced responsiveness. PLoS One. 2010; 5(9):e12798.

40. Modesti PA, Fortini A, Gensini GF, Vanni D, Prisco D, Abbate R. Human prostacyclin platelet receptors in diabetes mellitus. Thromb Res. 1991;63(5):541-548.

41. Ramirez MA, Borja NL. Epalrestat: an aldose reductase inhibitor for the treatment of diabetic neuropathy. Pharmacotherapy. 2008;28(5):646-655.

42. Hotta N, et al. Long-term clinical effects of epalrestat, an aldose reductase inhibitor, on diabetic peripheral neuropathy: the 3-year, multicenter, comparative Aldose Reductase Inhibitor-Diabetes Complications Trial. Diabetes Care. 2006;29(7):1538-1544.

43. Santilli F, et al. Postprandial hyperglycemia is a determinant of platelet activation in early type 2 diabetes mellitus. J Thromb Haemost. 2010;8(4):828-837.

44. Kajita K, et al. Increased platelet aggregation in diabetic patients with microangiopathy despite good glycemic control. Platelets. 2001;12(6):343-351.

45. Alessandrini P, McRae J, Feman S, FitzGerald GA. Thromboxane biosynthesis and platelet function in type I diabetes mellitus. $N$ Engl J Med. 1988; 319(4):208-212.

46. Saxena SP, Brandes LJ, Becker AB, Simons KJ, LaBella FS, Gerrard JM. Histamine is an intracellular messenger mediating platelet aggregation. Science. 1989; 243(4898):1596-1599.

47. Efendic S, Luft R, Wajngot A. Aspects of the pathogenesis of type 2 diabetes. Endocr Rev. 1984:5(3):395-410.

48. Theoret JF, Bienvenu JG, Kumar A, Merhi Y. P-selectin antagonism with recombinant $\mathrm{p}$-selectin glycoprotein ligand-1 (rPSGL-Ig) inhibits circulating activated platelet binding to neutrophils induced by damaged arterial surfaces.JPharmacol Exp Ther. 2001; 298(2):658-664.

49. Shinmura $\mathrm{K}$, et al. Aldose reductase is an obligatory mediator of the late phase of ischemic preconditioning. Circ Res. 2002;91(3):240-246.

50. Tsakiris DA, Scudder L, Hodivala-Dilke K, Hynes RO, Coller BS. Hemostasis in the mouse (Mus musculus): a review. Thromb Haemost. 1999;81(2):177-188.

51. Nunn B. Some characteristics of mouse platelet aggregation and a comparison of the activity of a range of compounds in mouse and human plateletrich plasma in vitro. Thromb Haemost. 1981;45(1):1-5.

52. Ciabattoni G, Pugliese F, Davi G, Pierucci A, Simonetti BM, Patrono C. Fractional conversion of thromboxane B2 to urinary 11-dehydrothromboxane B2 in man. Biochim Biophys Acta. 1989;992(1):66-70.

53. Gluckman TJ, et al. Effects of aspirin responsiveness and platelet reactivity on early vein graft thrombosis after coronary artery bypass graft surgery. J Am Coll Cardiol. 2011;57(9):1069-1077.

54. Gluckman TJ, et al. Effect of anti-platelet factor-4/ heparin antibody induction on early saphenous vein graft occlusion after coronary artery bypass surgery. J Thromb Haemost. 2009;7(9):1457-1464. 